䟚 


\section{Akten zur Auswärtigen Politik der Bundesrepublik Deutschland}

Herausgegeben im Auftrag des Auswärtigen Amts vom Institut für Zeitgeschichte

Hauptherausgeber

Hans-Peter Schwarz

Mitherausgeber

Helga Haftendorn, Klaus Hildebrand, Werner Link, Horst Möller und Rudolf Morsey

R. Oldenbourg Verlag München 2000 


\title{
Akten zur Auswärtigen Politik der Bundesrepublik Deutschland
}

\author{
1952
}

1. Januar bis 31. Dezember 1952

Wissenschaftlicher Leiter

Rainer A. Blasius

\author{
Bearbeiter \\ Martin Koopmann \\ und Joachim Wintzer
}

R. Oldenbourg Verlag München 2000 
Die Deutsche Bibliothek - CIP-Einheitsaufnahme

\begin{abstract}
Akten zur auswärtigen Politik der Bundesrepublik Deutschland / hrsg. im Auftr. des Auswärtigen Amts vom Institut für Zeitgeschichte. - München : Oldenbourg
\end{abstract}

1952. 1. Januar bis 31. Dezember 1952. - 2000

ISBN 3-486-56480-3

(C) 2000 Oldenbourg Wissenschaftsverlag GmbH, München Rosenheimer Straße 145, D-81671 München Internet: http://www.oldenbourg-verlag.de

Das Werk einschließlich aller Abbildungen ist urheberrechtlich geschützt. Jede Verwertung außerhalb der Grenzen des Urheberrechtsgesetzes ist ohne Zustimmung des Verlages unzulässig und strafbar. Dies gilt insbesondere für Vervielfältigungen, Übersetzungen, Mikroverfilmungen und die Einspeicherung und Bearbeitung in elektronischen Systemen.

Umschlaggestaltung: Dieter Vollendorf

Gedruckt auf säurefreiem, alterungsbeständigem Papier (chlorfrei gebleicht).

Gesamtherstellung: R. Oldenbourg Graphische Betriebe Druckerei GmbH, München

ISBN 3-486-56480-3 


\section{Inhalt}

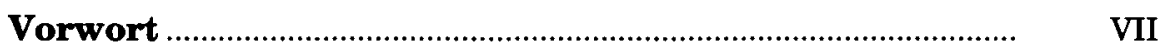

Vorbemerkungen zur Edition.................................................... VIII

Verzeichnisse

Dokumentenverzeichnis .................................................................. XVII

Literaturverzeichnis ...................................................................... XIVVIII

Abkürzungsverzeichnis .................................................................. LVII

Dokumente....................................................................................

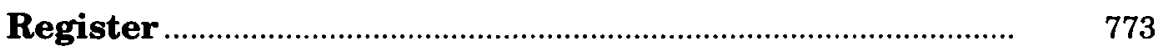

Personenregister ..............................................................................

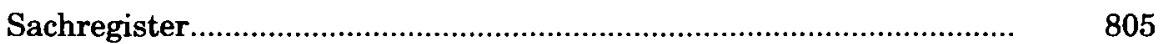

Organisationsplan des Auswärtigen Amts vom August 1952 …............ $\quad 843$ 



\section{Vorwort}

Mit den „Akten zur Auswärtigen Politik der Bundesrepublik Deutschland 1952“ wird die Publikation von Dokumenten aus dem Politischen Archiv des Auswärtigen Amts über die Jahre 1949 bis 1962 fortgesetzt. Der Band ergänzt die bereits 1990 unter meiner Verantwortung publizierte Edition „Adenauer und die Hohen Kommissare 1952“.

Das Erscheinen des vorliegenden Bandes gibt Anlaß, allen an dem Werk Beteiligten zu danken. So gilt mein verbindlichster Dank dem Auswärtigen Amt, insbesondere dem Politischen Archiv sowie den Damen und Herren in den Referaten, die beim Deklassifizierungsverfahren zur Offenlegung der Dokumente beigetragen haben. In gleicher Weise zu danken ist dem Bundesarchiv in Koblenz für die Erlaubnis, einige Aufzeichnungen aus dem Nachlaß des Botschafters a.D. Herbert Blankenhorn und aus dem Bestand Bundeskanzleramt (B 136) einbeziehen zu können, sowie dem Bundesarchiv/Militärarchiv in Freiburg im Breisgau und der Stiftung Bundeskanzler-Adenauer-Haus in Rhöndorf.

Besonderer Dank gebührt ferner den Kollegen im Herausgebergremium, die sich ihrer viel Zeit in Anspruch nehmenden Aufgabe in bewährter Kollegialität gewidmet haben. Ferner sei die tadellose Zusammenarbeit mit den zuständigen Persönlichkeiten und Gremien des Instituts für Zeitgeschichte dankbar hervorgehoben. Gedankt sei auch dem präzise arbeitenden Verlag R. Oldenbourg.

Das Hauptverdienst am Gelingen des Bandes gebührt den Bearbeitern, Herrn Dr. Martin Koopmann und Herrn Dr. Joachim Wintzer, zusammen mit dem Wissenschaftlichen Leiter, Herrn Dr. Rainer A. Blasius. Ihnen sei für die erbrachte Leistung nachdrücklichst gedankt.

Ebenso haben wesentlich zur pünktlichen Fertigstellung der Edition beigetragen: Herr Dr. Daniel Kosthorst durch die Vorarbeiten für die Dokumentensammlung, Herr Dr. Matthias Jaroch durch die Bearbeitung von drei Monaten des Jahres 1952 und Herr Dr. Wolfgang Hölscher durch die Beratung bei der Herstellung des Umbruchs.

Die „Akten zur Auswärtigen Politik der Bundesrepublik Deutschland 1953“ befinden sich in Arbeit.

Bonn, den 1. Februar 2000

Hans-Peter Schwarz 


\section{Vorbemerkungen zur Edition}

Die "Akten zur Auswärtigen Politik der Bundesrepublik Deutschland 1952“ (Kurztitel: AAPD 1952) umfassen 253 Dokumente. Den abgedruckten Dokumenten gehen neben Vorwort und Vorbemerkungen ein Dokumentenverzeichnis, ein Literaturverzeichnis sowie ein Abkürzungsverzeichnis voran. Am Ende des Bandes findet sich ein Personen- und ein Sachregister sowie ein Organisationsplan des Auswärtigen Amts vom August 1952.

\section{Dokumentenauswahl}

Grundlage für die Fondsedition der „Akten zur Auswärtigen Politik der Bundesrepublik Deutschland 1952“ sind die Bestände des Politischen Archivs des Auswärtigen Amts (PA/AA). Besonderes Gewicht wurde auf den Bestand der Politischen Abteilung gelegt. Angemessene Berücksichtigung fanden aber auch die anderen Abteilungen des Auswärtigen Amts, insbesondere die Länder-, die Rechts- und die Kulturabteilung. Über die Konferenz für die Organisation einer Europäischen Verteidigungsgemeinschaft gaben die Akten der „EVGDelegation" Aufschluß. Die Handakten des Leiters der Politischen Abteilung im Auswärtigen Amt, Herbert Blankenhorn, befinden sich zu einem Teil im PA/AA und zu einem anderen Teil im Bundesarchiv in Koblenz. Sie wurden ebenso ausgewertet wie einzelne Bestände aus dem Bundesarchiv-Militärarchiv in Freiburg und aus der Stiftung Bundeskanzler-Adenauer-Haus in Rhöndorf (Nachlaß Adenauer).

Entsprechend ihrer Herkunft belegen die edierten Dokumente in erster Linie die außenpolitischen Aktivitäten des Bundeskanzlers und Bundesministers des Auswärtigen. Die Rolle anderer Akteure, insbesondere im parlamentarischen und parteipolitischen Bereich, wird beispielhaft dokumentiert, sofern eine Wechselbeziehung zum Auswärtigen Amt gegeben war.

Die ausgewählten Dokumente ergänzen die bereits im Jahr 1990 publizierte Edition „Adenauer und die Hohen Kommissare 1952“. Erstmals werden Schriftstücke veröffentlicht, die bisher als Verschlußsachen der Geheimhaltung unterworfen waren. Dank einer entsprechenden Ermächtigung wurden den Bearbeitern die VS-Bestände des PA/AA ohne Einschränkung zugänglich gemacht und Anträge auf Herabstufung und Offenlegung von Schriftstücken beim Auswärtigen Amt ermöglicht. Kopien der offengelegten Schriftstücke, deren Zahl diejenige der in den AAPD 1952 edierten Dokumente weit übersteigt, werden im PA/AA zugänglich gemacht (Bestand B 150).

Nur eine äußerst geringe Zahl der für die Edition vorgesehenen Aktenstücke wurde nicht zur Veröffentlichung freigegeben. Hierbei handelt es sich vor allem um Dokumente, in denen personenbezogene Vorgänge im Vordergrund stehen oder die auch heute noch sicherheitsrelevante Angaben enthalten. Von einer Deklassifizierung ausgenommen war Schriftgut ausländischer Herkunft bzw. aus dem Bereich multilateraler oder internationaler Organisationen. Unberücksichtigt blieb ebenfalls nachrichtendienstliches Material. 
Zur Dokumentation wesentlicher historischer Vorgänge wurden in Ausnahmefällen bereits an anderer Stelle veröffentlichte Schriftstücke von besonderer Bedeutung in die Auswahl aufgenommen.

\section{Dokumentenfolge}

Die 253 edierten Dokumente sind in chronologischer Folge geordnet und mit laufenden Nummern versehen. Bei differierenden Datumsangaben auf einem Schriftstück, z.B. im Falle abweichender maschinenschriftlicher und handschriftlicher Datierung, ist in der Regel das früheste Datum maßgebend. Mehrere Dokumente mit demselben Datum sind, soweit möglich, nach der Uhrzeit eingeordnet. Erfolgt eine Datierung lediglich aufgrund sekundärer Hinweise (z.B. aus Begleitschreiben, beigefügten Vermerken usw.), wird dies in einer Anmerkung ausgewiesen. Bei Aufzeichnungen über Gespräche ist das Datum des dokumentierten Vorgangs ausschlaggebend, nicht der meist spätere Zeitpunkt der Niederschrift.

\section{Dokumentenkopf}

Jedes Dokument beginnt mit einem halbfett gedruckten Dokumentenkopf, in dem wesentliche formale Angaben zusammengefaßt werden. Auf Dokumentennummer und Dokumentenüberschrift folgen in kleinerer Drucktype ergänzende Angaben, so rechts außen das Datum. Links außen wird, sofern vorhanden, das Geschäftszeichen des edierten Schriftstücks einschließlich des Geheimhaltungsgrads (zum Zeitpunkt der Entstehung) wiedergegeben. Das Geschäftszeichen, das Rückschlüsse auf den Geschäftsgang zuläßt und die Ermittlung zugehörigen Aktenmaterials ermöglicht, besteht in der Regel aus der Kurzbezeichnung der ausfertigenden Arbeitseinheit sowie aus weiteren Elementen wie dem inhaltlich definierten Aktenzeichen, der Tagebuchnummer einschließlich verkürzter Jahresangabe und gegebenenfalls dem Geheimhaltungsgrad. Dokumentennummer, verkürzte Überschrift und Datum finden sich auch im Kolumnentitel über dem Dokument.

Den Angaben im Dokumentenkopf läßt sich die Art des jeweiligen Dokuments entnehmen. Aufzeichnungen sind eine in der Edition besonders häufig vertretende Dokumentengruppe. Der Verfasser wird jeweils in der Überschrift benannt, auch dann, wenn er sich nur indirekt erschließen läßt. Letzteres wird in einer Anmerkung vermerkt. Läßt sich ein solcher weder mittelbar noch unmittelbar nachweisen, wird die ausfertigende Arbeitseinheit (Abteilung, Referat oder Delegation) angegeben.

Breiten Raum nehmen Gesprächsaufzeichnungen sowie Niederschriften über Besprechungen und Konferenzen ein. Sie werden als solche in der Überschrift gekennzeichnet und chronologisch nach dem Gesprächs-, Besprechungs- oder Konferenzdatum eingeordnet, während Verfasser und Datum der Niederschrift - sofern ermittelbar - in einer Anmerkung ausgewiesen sind.

Eine wichtige Dokumentengruppe sind darüber hinaus Schreiben, erkennbar jeweils an der Nennung von Absender und Empfänger. Dazu gehören insbeson- 
dere die Schreiben des Bundeskanzlers an die Hohen Kommissare, die entweder an einen der Hohen Kommissare allein oder an den im monatlichen Wechsel amtierenden Geschäftsführenden Vorsitzenden der AHK gerichtet wurden. Ferner zählen dazu Schreiben der Bundesregierung, vertreten durch den Bundeskanzler und Bundesminister des Auswärtigen, an ausländische Regierungen, desgleichen auch Korrespondenz des Auswärtigen Amts mit anderen Ressorts oder mit Bundestagsabgeordneten.

Eine weitere Gruppe von Dokumenten bildet der Schriftverkehr zwischen der Zentrale in Bonn und den Auslandsvertretungen. Diese erhielten ihre Informationen und Weisungen in der Regel mittels Drahterlaß, der fernschriftlich oder per Funk übermittelt wurde. Auch bei dieser Dokumentengruppe wird in der Überschrift der Verfasser genannt, ein Empfänger dagegen nur, wenn der Drahterlaß an eine einzelne Auslandsvertretung bzw. deren Leiter gerichtet war. Anderenfalls werden die Adressaten in einer Anmerkung aufgeführt. Bei Runderlassen an sehr viele oder an alle diplomatischen Vertretungen wird der Empfängerkreis nicht näher spezifiziert, um die Anmerkungen nicht zu überfrachten. Ebenso sind diejenigen Auslandsvertretungen nicht eigens aufgeführt, die nur nachrichtlich von einem Erlaß in Kenntnis gesetzt wurden. Ergänzend zum Geschäftszeichen wird im unteren Teil des Dokumentenkopfes links die Nummer des Drahterlasses sowie der Grad der Dringlichkeit angegeben. Rechts davon befindet sich das Datum und - sofern zu ermitteln - die Uhrzeit der Aufgabe. Ein Ausstellungsdatum wird nur dann angegeben, wenn es vom Datum der Aufgabe abweicht.

Der Dokumentenkopf bei einem im Auswärtigen Amt eingehenden Drahtbericht ist in Analogie zum Drahterlaß gestaltet. Außer Datum und Uhrzeit der Aufgabe wird auch der Zeitpunkt der Ankunft festgehalten, jeweils in Ortszeit.

In weniger dringenden Fällen verzichteten die Botschaften auf eine fernschriftliche Übermittlung und zogen die Form des mit Kurier übermittelten Schriftberichts vor. Beim Abdruck solcher Stücke werden im Dokumentenkopf neben der Überschrift mit Absender und Empfänger das Geschäftszeichen und das Datum genannt.

Die wenigen Dokumente, die sich keiner der beschriebenen Gruppen zuordnen lassen, sind aufgrund individueller Überschriften zu identifizieren.

Die Überschrift bei allen Dokumenten enthält die notwendigen Angaben zum Ausstellungs-, Absende- oder Empfangsort bzw. zum Ort des Gesprächs. Erfolgt keine besondere Ortsangabe, ist stillschweigend Bonn zu ergänzen. Hält sich der Verfasser oder Absender eines Dokuments nicht an seinem Dienstort auf, wird der Ortsangabe ein „z.Z.“ vorangesetzt.

Bei den edierten Schriftstücken handelt es sich in der Regel jeweils um die erste Ausfertigung oder - wie etwa bei den Drahtberichten - um eines von mehreren gleichrangig nebeneinander zirkulierenden Exemplaren. Statt einer Erstausfertigung mußten gelegentlich ein Durchdruck, eine Abschrift, eine Ablichtung oder ein vervielfältigtes Exemplar (Matrizenabzug) herangezogen werden. Ein entsprechender Hinweis findet sich in einer Anmerkung. In wenigen Fällen sind Entwürfe abgedruckt und entsprechend in den Überschriften kenntlich gemacht. 


\section{Dokumententext}

Unterhalb des Dokumentenkopfes folgt - in normaler Drucktype - der Text des jeweiligen Dokuments, einschließlich des Betreffs, der Anrede und der Unterschrift. Die Dokumente werden ungekürzt veröffentlicht. Sofern in Ausnahmefällen Auslassungen vorgenommen werden müssen, ist dies kenntlich gemacht und in einer Anmerkung erläutert. Textergänzungen der Bearbeiter stehen in eckigen Klammern.

Offensichtliche Schreib- und Interpunktionsfehler werden stillschweigend korrigiert. Eigentümliche Schreibweisen bleiben nach Möglichkeit erhalten; im Bedarfsfall wird jedoch vereinheitlicht bzw. modernisiert. Dies trifft teilweise auch auf fremdsprachige Orts- und Personennamen zu, deren Schreibweise nach den im Auswärtigen Amt gebräuchlichen Regeln wiedergegeben wird.

Selten vorkommende und ungebräuchliche Abkürzungen werden in einer Anmerkung aufgelöst. Typische Abkürzungen von Institutionen, Parteien etc. werden allerdings übernommen. Hervorhebungen in der Textvorlage, also etwa maschinenschriftliche Unterstreichungen oder Sperrungen, werden nicht wiedergegeben. Der Kursivdruck dient dazu, bei Gesprächsaufzeichnungen die Sprecher voneinander abzuheben. Im äußeren Aufbau (Absätze, Überschriften usw.) folgt das Druckbild nach Möglichkeit der Textvorlage.

Unterschriftsformeln werden vollständig wiedergegeben. Ein handschriftlicher Namenszug ist nicht besonders gekennzeichnet, eine Paraphe mit Unterschriftscharakter wird aufgelöst (mit Nachweis in einer Anmerkung). Findet sich auf einem Schriftstück der Name zusätzlich maschinenschriftlich vermerkt, bleibt dies unerwähnt. Ein maschinenschriftlicher Name, dem ein "gez." vorangestellt ist, wird entsprechend übernommen; fehlt in der Textvorlage der Zusatz "gez.", wird er in eckigen Klammern ergänzt. Weicht das Datum der Paraphe vom Datum des Schriftstückes ab, wird dies in der Anmerkung ausgewiesen.

Unter dem Dokumententext wird die jeweilige Fundstelle des Schriftstückes in halbfetter Schrifttype nachgewiesen. Bei Dokumenten aus dem PA/AA wird auf die Angabe des Archivs verzichtet und nur der jeweilige Bestand mit Bandnummer genannt. Dokumente aus VS-Beständen sind mit der Angabe „VS-Bd.“ versehen. Bei Dokumenten anderer Herkunft werden Archiv und Bestandsbezeichnung angegeben. Liegt ausnahmsweise ein Schriftstück bereits veröffentlicht vor, so wird dies in einer gesonderten Anmerkung nach der Angabe der Fundstelle ausgewiesen.

\section{Kommentierung}

In Ergänzung zum Dokumentenkopf enthalten die Anmerkungen formale Hinweise und geben Auskunft über wesentliche Stationen im Geschäftsgang. Angaben technischer Art, wie Registraturvermerke oder standardisierte Verteiler, werden nur bei besonderer Bedeutung erfaßt. Wesentlich ist dagegen die Frage, welche Beachtung das jeweils edierte Dokument gefunden hat. Dies läßt sich an den Paraphen maßgeblicher Akteure sowie an den - überwiegend handschriftlichen - Weisungen, Bemerkungen oder auch Reaktionen in Form von Frage- oder Ausrufungszeichen ablesen, die auf dem Schriftstück selbst oder 
auf Begleitschreiben und Begleitvermerken zu finden sind. Die diesbezüglichen Merkmale sowie damit in Verbindung stehende Hervorhebungen (Unterstreichungen oder Anstreichungen am Rand) werden in Anmerkungen nachgewiesen. Auf den Nachweis sonstiger An- oder Unterstreichungen wird verzichtet. Abkürzungen in handschriftlichen Passagen werden in eckigen Klammern aufgelöst, sofern sie nicht im Abkürzungsverzeichnis aufgeführt sind.

In den im engeren Sinn textkritischen Anmerkungen werden nachträgliche Korrekturen oder textliche Änderungen des Verfassers und einzelner Adressaten festgehalten, sofern ein Konzipient das Schriftstück entworfen hat. Unwesentliche Textverbesserungen sind hiervon ausgenommen. Ferner wird auf einen systematischen Vergleich der Dokumente mit Entwürfen ebenso verzichtet wie auf den Nachweis der in der Praxis üblichen Einarbeitung von Textpassagen in eine spätere Aufzeichnung oder einen Drahterlaß.

Die Kommentierung soll den historischen Zusammenhang der edierten Dokumente in ihrer zeitlichen und inhaltlichen Abfolge sichtbar machen, weiteres Aktenmaterial und anderweitiges Schriftgut nachweisen, das unmittelbar oder mittelbar angesprochen wird, sowie Ereignisse oder Sachverhalte näher erläutern, die dem heutigen Wissens- und Erfahrungshorizont ferner liegen und aus dem Textzusammenhang heraus nicht oder nicht hinlänglich zu verstehen sind.

Besonderer Wert wird bei der Kommentierung darauf gelegt, die Dokumente durch Bezugsstücke aus den Akten der verschiedenen Arbeitseinheiten des Auswärtigen Amts bis hin zur Leitungsebene zu erläutern. Zitate oder inhaltliche Wiedergaben sollen die Entscheidungsprozesse erhellen und zum Verständnis der Dokumente beitragen. Dadurch wird zugleich Vorarbeit geleistet für eine vertiefende Erschließung der Bestände des PA/AA. Um die Identifizierung von Drahtberichten bzw. -erlassen zu erleichtern, werden außer dem Verfasser und dem Datum die Drahtberichtsnummer und, wo immer möglich, die Drahterlaßnummer angegeben.

Findet in einem Dokument veröffentlichtes Schriftgut Erwähnung - etwa Abkommen, Gesetze, Reden oder Presseberichte -, so wird die Fundstelle nach Möglichkeit genauer spezifiziert. Systematische Hinweise auf archivalische oder veröffentlichte Quellen, insbesondere auf weitere Bestände des PA/AA, erfolgen nicht. Sekundärliteratur wird generell nicht in die Kommentierung aufgenommen.

Angaben wie Dienstbezeichnung, Dienststellung, Funktion, Dienstbehörde und Nationalität dienen der eindeutigen Identifizierung der in der Kommentierung vorkommenden Personen. Bei Bundesministern erfolgt ein Hinweis zum jeweiligen Ressort nur im Personenregister. Eine im Dokumententext lediglich mit ihrer Funktion genannte Person wird nach Möglichkeit in einer Anmerkung namentlich nachgewiesen. Davon ausgenommen sind der jeweilige Bundespräsident, Bundeskanzler und Bundesminister des Auswärtigen.

Die Bezeichnung einzelner Staaten wird so gewählt, daß Verwechslungen ausgeschlossen sind. Als Kurzform für die Deutsche Demokratische Republik kommen in den Dokumenten die Begriffe SBZ oder DDR vor und werden so wiedergegeben. Der in der Forschung üblichen Praxis folgend, wird jedoch in der Kommentierung, den Verzeichnissen sowie den Registern der Begriff DDR verwen- 
det. Das Adjektiv „deutsch“ findet nur bei gesamtdeutschen Belangen oder dann Verwendung, wenn eine eindeutige Zuordnung gegeben ist. Der westliche Teil von Berlin wird als Berlin (West), der östliche Teil der Stadt als Ost-Berlin bezeichnet.

Für häufig benutzte Publikationen wie Editionen, Geschichtskalender und Memoiren werden Kurztitel oder Kurzformen eingeführt, die sich über ein entsprechendes Verzeichnis auflösen lassen. Der Platzersparnis dienen ebenfalls die Rückverweise auf bereits an anderer Stelle ausgeführte Anmerkungen. Häufig genannte Verträge oder Gesetzestexte werden nur bei der Erstnennung nachgewiesen und lassen sich über das Sachregister erschließen.

Wie bei der Wiedergabe der Dokumente finden auch in den Anmerkungen die im Auswärtigen Amt gebräuchlichen Regeln für die Transkription fremdsprachlicher Namen und Begriffe Anwendung. Bei Literaturangaben in russischer Sprache wird die im wissenschaftlichen Bereich übliche Transliterierung durchgeführt.

\section{Verzeichnisse}

Das Dokumentenverzeichnis ist chronologisch angelegt. Es bietet zu jedem Dokument folgende Angaben: Die halbfett gedruckte Dokumentennummer, Datum und Überschrift, die Fundseite sowie eine inhaltliche Kurzübersicht.

Das Literaturverzeichnis enthält nur solche Publikationen, die häufig zur Kommentierung herangezogen und mit Kurztiteln oder Kurzformen versehen wurden. Diese sind alphabetisch geordnet und werden durch bibliographische Angaben aufgelöst.

Das Abkürzungsverzeichnis führt die im Dokumententeil vorkommenden Abkürzungen auf, insbesondere von Firmen, Organisationen, Parteien und Dienstbezeichnungen sowie sonstige im diplomatischen Schriftverkehr übliche Abbreviaturen. Nicht aufgenommen werden geläufige Abkürzungen wie ${ }_{n}$ z.B. ${ }^{*}$, „d.h. ${ }^{*}$, „m. E.“, „u.U.“ und „usw." sowie Abkürzungen, die im Dokumententext oder in einer Anmerkung erläutert sind.

\section{Register und Organisationsplan}

Im Personenregister werden in der Edition vorkommende Personen unter Nennung derjenigen politischen, dienstlichen oder beruflichen Funktionen aufgeführt, die im inhaltlichen Zusammenhang der Dokumente wesentlich sind. Das Sachregister ermöglicht einen thematisch differenzierten Zugriff auf die einzelnen Dokumente. Näheres ist den Hinweisen zur Benutzung des jeweiligen Registers auf S. 773 und S. 805 zu entnehmen.

Der Organisationsplan vom August 1952 zeigt die Struktur des Auswärtigen Amts und informiert über die Namen der Leiter der jeweiligen Arbeitseinheiten. 

Verzeichnisse 



\section{Dokumentenverzeichnis}

Die Sitzung dient der Vorbereitung,der Konferenz über deutsche Auslandsschulden in London. Erörtert werden der Zeitpunkt des Konferenzbeginns sowie organisatorische Fragen. amerikanischen Hohen Kommissar McCloy

Im Mittelpunkt stehen Fragen der Organisation einer Europäischen Verteidigungsgemeinschaft, Beschränkungen der Rüstungsproduktion und der finanzielle Verteidigungsbeitrag der Bundesrepublik.

Hallstein faßt ein Gespräch mit dem französischen Stellvertretenden Hohen Kommissar Bérard über Beschränkungen der Rüstungsproduktion der Bundesrepublik zusammen.

Wever gibt eine Hausbesprechung wieder, in der Staatssekretär Hallstein von der Ministerkonferenz über eine Europäische Verteidigungsgemeinschaft in Paris berichtete. Themen der Konferenz waren die Organisation der Gemeinschaft, das Verhältnis der EVG zur europäischen Integration sowie das Gemeinschaftsbudget.

Jansen informiert über Äußerungen des luxemburgischen $\mathrm{Au}$ Benministers Bech zur EVG.

Strohm vermerkt ein Gespräch mit dem Mitarbeiter im britischen Hochkommissariat, Warr, über die Rückgabe Helgolands an die Bundesrepublik sowie über die Zuweisung von Ersatzzielen an die britische Luftwaffe.

Adenauer betont die Notwendigkeit, die Höhe des finanziellen Verteidigungsbeitrags der Bundesrepublik zwischen der Bundesrepublik und dem Temporary Council Committee zu erörtern. 
8

09.01. Besprechung mit Vertretern der Alliierten Hohen Kommission

Themen sind die Höhe des finanziellen Verteidigungsbeitrags der Bundesrepublik sowie die Frage, ob die Vertreter der Drei Mächte als Beauftragte des Temporary Council Committee oder der AHK fungieren.

9

10.01. Generalkonsul I. Klasse Krekeler, Washington, an das Auswärtige Amt

Krekeler informiert über ein Gespräch mit dem Abteilungsleiter im amerikanischen Außenministerium. Byroade berichtete, daß Premierminister Churchill bei seinem USA-Besuch die Unterstützung für eine Europäische Verteidigungsgemeinschaft zugesagt habe.

10.01. Runderlaß des Legationsrats I. Klasse Trützschler von Falkenstein

Trützschler informiert über die Haltung der USA zum Beitritt der Bundesrepublik zur NATO.

11

11.01. Aufzeichnung des Legationssekretärs a.D. Hess

Hess thematisiert die Durchführung der Zahlungs- und Kreditabkommen mit Jugoslawien.

12

11.01. Generalkonsul I. Klasse Krekeler, Washington, an das Auswärtige Amt

Krekeler berichtet von einer Unterredung mit dem Abteilungsleiter im amerikanischen Außenministerium, Byroade, über den Besuch des Präsidenten der evangelischen Kirche in Hessen und Nassau, Niemöller, in Moskau.

13

11.01. Generalkonsul I. Klasse Krekeler, Washington, an das Auswärtige Amt

Krekeler faßt ein Gespräch mit dem Abteilungsleiter im amerikanischen Außenministerium, Byroade, über die Haltung der Bundesregierung zum finanziellen Verteidigungsbeitrag der Bundesrepublik zusammen.

14
S. 24

\section{Blank}

Blank informiert über ein Gespräch mit dem amerikanischen Hohen Kommissar McCloy über den finanziellen Verteidigungsbeitrag der Bundesrepublik, Beschränkungen der Rüstungsproduktion sowie die territoriale Organisation einer Europäischen Verteidigungsgemeinschaft. 

Kommission

Gegenstand ist der finanzielle Verteidigungsbeitrag der Bundesrepublik.

Delegationsleiter Rendel

Abs erklärt die Bereitschaft der Bundesregierung, auf der Konferenz über deutsche Auslandsschulden neben den Nachkriegsverpflichtungen auch die Vorkriegsschulden unter Berücksichtigung der deutschen Transferfähigkeit zu behandeln.

Adenauer weist Hallstein an, die Behandlung der Saarfrage im Ministerkomitee des Europarats gründlich vorzubereiten.

Hallstein berichtet über ein Gespräch mit dem amerikanischen Hohen Kommissar. McCloy unterbreitete einen Vorschlag zur Verhandlungsführung bei den Besprechungen über den finanziellen Verteidigungsbeitrag der Bundesrepublik.

Grewe erläutert den Vorschlag, die Übernahme von Bundesgesetzen in Berlin (West) durch ein generelles Mantelgesetz des Abgeordnetenhauses zu regeln. Kommission

Im Mittelpunkt stehen die Zahlungsmodalitäten des finanziellen Verteidigungsbeitrags der Bundesrepublik. Kommission

Die Delegation der Bundesrepublik unterbreitet einen Vorschlag zum finanziellen Verteidigungsbeitrag der Bundesrepublik.

Adenauer teilt mit, daß die Bundesregierung ein Embargo für die Lieferung von Gütern mit strategischer Bedeutung an osteuropäische Staaten anordnen werde. 
23

24.01. Bundeskanzler Adenauer an den Geschäftsführenden Vorsitzenden der Alliierten Hohen Kommission, Kirkpatrick

Adenauer tritt für beschleunigte Verhandlungen über die Ablösung des Besatzungsstatuts ein.

26.01. Aufzeichnung des Vortragenden Legationsrats Salat

Salat berichtet von einer Besprechung mit den Kultusministern der Länder sowie den Kulturbeauftragten der AHK über die kulturellen Beziehungen zwischen der Bundesrepublik und den Drei Mächten.

26.01. Außenministerkonferenz über eine Europäische Verteidigungsgemeinschaft in Paris

Erörtert werden die Zusammensetzung der künftigen Gemeinschaftsorgane und die Stimmengewichtung im Ministerrat.

27.01. Aufzeichnung des Vortragenden Legationsrats von Kessel, Paris

Kessel notiert eine Weisung des Bundeskanzlers Adenauer, daß der Beitritt der Bundesrepublik zur NATO auf der Außenministerkonferenz über eine Europäische Verteidigungsgemeinschaft in Paris vorrangig zu behandeln sei. Verteidigungsgemeinschaft in Paris

Themen sind die Errichtung einer europäischen Föderation auf der Grundlage der Europäischen Verteidigungsgemeinschaft sowie die Zusammensetzung der EVG-Organe und die Stimmengewichtung im Ministerrat.

Blankenhorn berichtet von einem Gespräch mit dem Politischen Berater im amerikanischen Hochkommissariat, Reber, über die Verleihung des persönlichen Titels eines Botschafters an den französischen Hohen Kommissar für die Saar, Grandval. Verteidigungsgemeinschaft in Paris

Staatssekretär Hallstein erklärt, daß die Bundesrepublik trotz der Gründung der EVG nicht auf eine gleichberechtigte Mitgliedschaft in der NATO verzichten könne.

Vialon analysiert den Verlauf der Außenministerkonferenz am 26. und 27. Januar 1952 in Paris über eine Europäische Verteidigungsgemeinschaft. 
Strohm skizziert wesentliche Aspekte der französischen Saarpolitik. amerikanischen Hohen Kommissar McCloy in München

Thema ist die Verleihung des persönlichen Titels eines Botschafters an den französischen Hohen Kommissar für die Saar, Grandval.

Ophüls faßt ein Gespräch mit dem Vorsitzenden der EVG-Konferenz, Alphand, über das Verhältnis zwischen EVG, Bundesrepublik und NATO zusammen.

Böker informiert über Meinungsverschiedenheiten bei den Vorbereitungen zur Einsetzung der UNO-Kommission für gesamtdeutsche Wahlen.

Krekeler berichtet über ein Gespräch mit dem Abteilungsleiter im amerikanischen Außenministerium, Byroade, zum Verhältnis der Bundesrepublik zur NATO und zur Verleihung des persönlichen Titels eines Botschafters an den französischen Hohen Kommissar für die Saar, Grandval. Staatssekretär Hallstein

Krekeler informiert über die Verhandlungen von Finanzexperten mit Vertretern des Internationalen Währungsfonds und der Weltbank in Washington über einen Beitritt der Bundesrepublik.

Hallstein erläutert den Wunsch nach einem NATO-Beitritt der Bundesrepublik.

Du Mont berichtet über ein Gespräch mit dem niederländischen Außenminister. Stikker äußerte sich zu einem NATO-Beitritt der Bundesrepublik und zur französischen Saarpolitik. 
Grewe erörtert den Entwurf der Drei Mächte vom 22. Dezember 1951 für den die Rechte und Pflichten der Streitkräfte betreffenden Teil des Truppenabkommens.

Böker faßt eine Ressortbesprechung über die Vorbereitungen der Wiedergutmachungsverhandlungen mit Israel und den jüdischen Organisationen zusammen. Auswärtige Amt

Krekeler berichtet von einem Gespräch mit der amerikanischen Regierung über die Auswirkungen der Saarfrage auf die NATOMinisterratstagung in Lissabon und die Teilnahme der Bundesrepublik an der Londoner Außenministerkonferenz.

Plehwe resümiert eine Ressortbesprechung über den von der AHK angeregten Beitritt der Bundesrepublik zum Weltpostverein. Auswärtige Amt

Krekeler informiert über ein Gespräch mit dem Abteilungsleiter im amerikanischen Außenministerium, Byroade. Im Mittelpunkt stand ein Beitritt der Bundesrepublik zur NATO. Kommissar Kirkpatrick

Adenauer legt rechtliche Möglichkeiten dar, um die Anwerbung ehemaliger Wehrmachtsangehöriger durch die ägyptische Regierung zu unterbinden.

Der Verlauf eines Gesprächs der Bundesminister Blücher, Erhard und Schäffer mit den Drei Weisen in Paris über den finanziellen Verteidigungsbeitrag der Bundesrepublik wird wiedergegeben.

Much plädiert für eine Generalzuständigkeit des Auswärtigen Amts in den mit der EGKS zusammenhängenden Fragen. 

mit dem Vorsitzenden der EVG-Konferenz, Alphand, in Paris

Blank und Alphand erörtern die Regelung der Beziehungen zwischen der EVG und der NATO.

Hallstein vermerkt den Gang einer Unterredung des Bundeskanzlers Adenauer mit dem amerikanischen Hohen Kommissar McCloy. Themen waren das Verhältnis zwischen EVG und NATO, die Saarfrage, die Behandlung der von Militärgerichten verurteilten Deutschen, der finanzielle Verteidigungsbeitrag der Bundesrepublik sowie die politische Lage in Frankreich.

Mosler berichtet vom Sachstand der Verhandlungen mit der AHK über die Einbeziehung von Berlin (West) in internationale Abkommen der Bundesrepublik und bittet um weitere Weisung. Zürich

Erörtert werden Liquiditätsprobleme der Europäischen Zahlungsunion und Perspektiven für eine Reform der EZU. London

Böker faßt ein Gespräch mit dem Vorsitzenden des World Jewish Congress, Goldmann, über die Vorbereitungen der Wiedergutmachungsverhandlungen zusammen.

Themen sind die Behandlung der von Militärgerichten verurteilten Deutschen sowie der finanzielle Verteidigungsbeitrag der Bundesrepublik.

Besprochen werden das Verhältnis der EVG zur NATO, die Behandlung der von Militärgerichten verurteilten Deutschen sowie die von den Drei Mächten angestrebte Erklärung der Bundesregierung über den Verzicht auf die Produktion bestimmter Rüstungsgüter.

Etzdorf empfiehlt die Errichtung diplomatischer Vertretungen in Portugal und Spanien. 


\subsection{Außenministerkonferenz in London}

Im Mittelpunkt stehen die Beschränkungen der Rüstungsproduktion der Bundesrepublik.

20.02. Staatssekretär Hallstein an den Beauftragten des Bundeskanzlers, Blank

Hallstein bittet Blank, die Positionen zu verschiedenen offenen Punkten für die weiteren Verhandlungen bei der EVG-Konferenz in Paris vorzubereiten. Kommissar François-Poncet

Hallstein äußert sich zur geplanten Verpachtung von Steinkohlefeldern im Saargebiet an die Charbonnages de France. Bundeskanzleramt

Pühl berichtet von einem Gespräch mit Vertretern des Bundesministeriums der Justiz über die Suspendierung einer Ausfuhrgenehmigung für Sprengstoff nach Ägypten. DIHT, Wenhold, an Bundeskanzler Adenauer

Wenhold empfiehlt die Errichtung amtlicher Wirtschaftsvertretungen in Afrika sowie im Nahen und Mittleren Osten. von Falkenstein, z.Z. Genf

Trützschler berichtet über ein Gespräch mit dem Vorsitzenden der UNO-Kommission für gesamtdeutsche Wahlen, Viana, zur Tätigkeit der Kommission in Deutschland. Außenminister Acheson

Adenauer dankt den USA für ihre Haltung bei den Verhandlungen über den finanziellen Verteidigungsbeitrag der Bundesrepublik. chardson (SHAPE) zusammen. Im Mittelpunkt standen die Ergebnisse der NATO-Ministerratstagung in Lissabon. 
Nostitz-Drzewiecki resümiert ein Gespräch mit dem österreichischen Generalkonsul Schöner über die Errichtung einer inoffiziellen Vertretung in Wien.

Böker berichtet über ein Gespräch mit dem Vorsitzenden der europäischen Sektion des World Jewish Congress, Barou, zu organisatorischen Fragen der Wiedergutmachungsverhandlungen sowie zum Umfang der israelischen Forderungen.

Grewe problematisiert die Frage der Zuständigkeit der Drei Mächte für die Vernehmung von Staatsangehörigen der UdSSR, die in die Bundesrepublik geflüchtet sind.

Hallstein unterbreitet eine Kabinettsvorlage zu Verhandlungen mit den Niederlanden über die Grenzfrage.

Mosler berichtet über ein Gespräch mit dem Abteilungsleiter im amerikanischen Außenministerium, Noble, zur Rückgabe und Auswertung der Akten des Auswärtigen Amts aus der Zeit vor 1945.

Hallstein informiert die Auslandsvertretungen über Verstöße gegen die Konvention zum Schutze der Menschenrechte und Grundfreiheiten im Saargebiet sowie über eine Beschwerde der Bundesregierung beim Europarat. Vorsitzenden der Alliierten Hohen Kommission, François-Poncet

Adenauer erkundigt sich, wie die Drei Mächte auf die jüngsten deutschlandpolitischen Vorstöße der DDR und der UdSSR vom Februar 1952 reagieren wollen.

Salat berichtet über die Kulturbeziehungen zu den USA.

Speidel resümiert Ausführungen des französischen militärischen Chefdelegierten bei der EVG-Konferenz, de Larminat, über den Beitrag der militärischen Chefdelegierten zum Fortgang der EVG-Konferenz. 
Die Verhandlungen über Wiedergutmachungsleistungen an Is rael und die jüdischen Organisationen werden vorbereitet und mit den Positionen bei der Konferenz über deutsche Auslandsschulden abgestimmt. Staatssekretär Lenz, Bundeskanzleramt

Klein bittet die Bundesregierung, sich bei den Drei Mächten für Verhandlungen über die alliierten Vorbehaltsrechte in Berlin einzusetzen.

11.03. Aufzeichnung des Ministerialdirektors Blankenhorn

Blankenhorn erörtert die sowjetische Note vom 10. März 1952.

11.03. Staatssekretär Hallstein, z.Z. Washington, an Bundeskanzler Adenauer

Hallstein berichtet von einem Gespräch mit dem amerikanischen Außenminister Acheson und dem Abteilungsleiter im amerikanischen Außenministerium, Byroade, über die sowjetische Note vom 10. März 1952 und die Saarfrage.

12.03. Vortragender Legationsrat von Kessel, Paris, an das Auswärtige Amt

Kessel übermittelt Äußerungen des Vorsitzenden der EVG-Konferenz, Alphand, zur sowjetischen Note vom 10. März 1952.

\subsection{Botschafter Du Mont, Den Haag, an das Auswärtige} Amt

Du Mont faßt ein Gespräch mit dem niederländischen Außenminister Stikker über die sowjetische Note vom 10. März 1952 zusammen.

12.03. Bundeskanzler Adenauer an Staatssekretär Hallstein, z.Z. Washington

Adenauer weist Hallstein an, sich künftig vor außenpolitischen Stellungnahmen mit ihm abzustimmen.

14.03. Staatssekretär Hallstein, z.Z. Washington, an Bundeskanzler Adenauer

Hallstein berichtet über ein Gespräch mit dem stellvertretenden Abteilungsleiter im amerikanischen Außenministerium, Lewis, zur Saarfrage und zur sowjetischen Note vom 10. März 1952.
S. 216 
Die Bundesregierung erklärt sich bereit, die von der UNO-Kommission für gesamtdeutsche Wahlen aufgestellten Bedingungen für deren Tätigkeit in Deutschland zu erfüllen. Außenminister Schuman

Adenauer übermittelt Schuman den Vorschlag einer Europäisierung des Saargebiets sowie den Entwurf einer Erklärung zur Saarfrage.

82 20./21. Gespräche des Bundeskanzlers Adenauer mit

03. Vertretern der Drei Mächte in Paris

Thema ist die Antwort der Drei Mächte auf die sowjetische Note vom 10. März 1952.

Adenauer faßt ein Gespräch mit dem französischen Außenminister Schuman über die Saarfrage zusammen.

Amt

Du Mont berichtet, daß der ägyptische Gesandte in Den Haag, Sadek Bey, Ansprüche der Arabischen Liga auf einen Teil der Wiedergutmachungsleistungen an Israel erhoben habe.

Blankenhorn gibt eine Mitteilung des Politischen Beraters im amerikanischen Hochkommissariat, Reber, zur Beantwortung der sowjetischen Note vom 10. März 1952 weiter.

\section{Bittenfeld}

Der Verlauf eines Gesprächs des Bundeskanzlers Adenauer mit dem britischen Außenminister Eden in Paris wird wiedergegeben. Themen waren die Saarfrage, die Unterzeichnung des Generalvertrages und die EVG. Amt

Richter informiert über Bestrebungen der DDR, eine Vertretung in Indien zu errichten. 
88

28.03. Aufzeichnung des Vortragenden Legationsrats von Kessel, Paris

Kessel erörtert Fragen einer Beistandsgarantie zwischen den EVG-Mitgliedstaaten sowie einer "Hilfeleistungsverpflichtung" Großbritanniens gegenüber der EVG.

89

28.03. Aufzeichnung des Vortragenden Legationsrats von Kessel, Paris

Kessel faßt ein Gespräch mit dem britischen Gesandten Hayter über die sowjetische Note vom 10. März 1952 zusammen.

29.03. Aufzeichnung des Beauftragten des Bundeskanzlers, Blank

Blank berichtet, wie die DDR und die Sowjetische Kontrollkommission in Deutschland die sowjetische Note vom 10. März 1952 und ihre Folgen beurteilen.

91

31.03. Delegationsleiter Abs, London, an Bundeskanzler Adenauer

Abs weist darauf hin, daß eine frühzeitige Anerkennung der Forderungen Israels bei den Wiedergutmachungsverhandlungen zu einem Scheitern der Konferenz über deutsche Auslandsschulden führen könne. Stellvertretenden Delegationsleiters Küster

Böhm und Küster ersuchen darum, zu den israelischen Forderungen bei den Wiedergutmachungsverhandlungen Stellung nehmen zu dürfen.

Blankenhorn informiert über die Tätigkeit der UNO-Kommission für gesamtdeutsche Wahlen und legt die Haltung der Bundesregierung dar.

94 04.04. Aufzeichnung der Delegation bei der EVG-Konferenz, Paris

Nach Darlegung der französischen Forderung nach Stimmrechtsparität zwischen der Bundesrepublik und Frankreich wird um Weisung gebeten. Adenauer

Gegenstand ist die Reaktion der Bundesregierung auf die israelischen Forderungen bei den Wiedergutmachungsverhandlungen. 
Kordt berichtet über die bevorstehende Aufnahme diplomatischer Beziehungen mit Japan.

Eine Besprechung mit der israelischen Delegation bei den Wiedergutmachungsverhandlungen über deren Forderungen wird zusammengefaßt.

Blankenhorn berichtet von einem Gespräch mit dem italienischen Botschafter Babuscio Rizzo über ein Handelsabkommen mit Italien. Auswärtige Amt

Kessel berichtet über die britische Zusage, eine Beistandsgarantie gegenüber der EVG abzugeben.

100 09.04. Aufzeichnung des Generalleutnants a.D. Speidel, Paris

Speidel faßt ein Gespräch mit dem Stabschef beim Oberbefehlshaber der alliierten Streitkräfte in Europa (SACEUR), Gruenther, zusammen. Themen waren die territoriale Organisation der EVG, die Dislozierung der EVG-Truppen in der Bundesrepublik, die sowjetische Note vom 10. März 1952 sowie die Behandlung der von Militärgerichten verurteilten Deutschen.

101 09.04. Aufzeichnung des Oberstleutnants a.D. de Maizière

De Maizière berichtet über eine Besprechung des Ausschusses für Industriebeschränkung zur Begrenzung der Rüstungsproduktion der Bundesrepublik.

102 10.04. Aufzeichnung des Obersten a. D. Graf von Kielmansegg

Kielmansegg informiert Ministerialdirektor Blankenhorn über die Verhandlungen der EVG-Konferenz am 8./9. April 1952.

103 10.04. Botschafter Du Mont, Den Haag, an Legationsrat I. Klasse Trützschler von Falkenstein

Du Mont berichtet, daß das niederländische Außenministerium auf eine Regelung der Grenzfrage dränge.

104 11.04. Generalkonsul I. Klasse Krekeler, Washington, an das Auswärtige Amt

Krekeler gibt eine Einschätzung der sowjetischen Note vom 9. April 1952 durch das amerikanische Außenministerium wieder. 
105 11.04. Aufzeichnung des Ministerialdirektors Blankenhorn

Blankenhorn skizziert die Position der Bundesregierung zur sowjetischen Note vom 9. April 1952.

12.04. Drahterlaß des Legationsrats I. Klasse Trützschler von Falkenstein

Trützschler weist die Auslandsvertretungen in London und $\mathrm{Pa}$ ris an, sich bei den Gastregierungen für eine rasche Aufnahme der Bundesrepublik in den Internationalen Währungsfonds und die Weltbank einzusetzen.

Meyer berichtet über Gespräche mit Ministerpräsident Nehru und hohen indischen Beamten. Hauptthemen waren die Stellung Indiens im internationalen System sowie die bilateralen Beziehungen.

108 16.04. Bundesminister Erhard an Bundeskanzler Adenauer

Erhard erörtert den Zusammenhang zwischen der Konferenz über deutsche Auslandsschulden und den Wiedergutmachungsverhandlungen.

109 18.04. Aufzeichnung des Ministerialdirektors Blankenhorn

Blankenhorn vermerkt eine Besprechung des Bundeskanzlers Adenauer und des Bundesministers Schäffer mit den Delegationsleitern bei den Wiedergutmachungsverhandlungen und der Konferenz über deutsche Auslandsschulden, Böhm und Abs.

110 19.04. Gespräch des Bundeskanzlers Adenauer mit dem Vorsitzenden des World Jewish Congress, Goldmann

Thema ist der Zusammenhang zwischen der Konferenz über deutsche Auslandsschulden und den Wiedergutmachungsverhandlungen.

111 22.04. Delegationsleiter Abs an Bundeskanzler Adenauer

Abs nimmt zum Schreiben des Bundesministers Erhard vom 16. April 1952 an Adenauer Stellung.

112 23.04. Runderlaß des Ministerialdirektors Blankenhorn

Blankenhorn informiert die Auslandsvertretungen über die Haltung zur sowjetischen Note vom 9. April 1952.

Kossmann legt Grundlinien einer neuen Ostpolitik der Bundesrepublik dar. 
114 25.04. Aufzeichnung des Legationsrats I. Klasse, Thierfelder

Thierfelder erörtert den Vorschlag des britischen Außenministers Eden, die Organe von EGKS, EVG und Europarat zusammenzulegen.

Haas erörtert Stellungnahmen von Mitgliedern des Untersuchungsausschusses Nr. 47 des Bundestages zur Personalpolitik des Auswärtigen Amts.

116 25.04. Besprechung des Ausschusses für Industriebeschränkung

Thema ist die Begrenzung der Herstellung von ferngelenkten Raketen und Kernbrennstoff durch die Bundesrepublik.

117 25.04. Botschafter Du Mont, Den Haag, an das Auswärtige Amt

Du Mont berichtet über ein Gespräch mit dem Generalsekretär des niederländischen Außenministeriums, Boon, über die sowjetische Note vom 9. April 1952. Auswärtige Amt

Krekeler teilt mit, daß die USA sowjetische Maßnahmen gegen Berlin anläßlich der Unterzeichnung des EVG- und des Generalvertrages befürchteten.

119 28.04. Generalkonsul I. Klasse Krekeler, Washington, an das Auswärtige Amt

Krekeler berichtet von dem amerikanischen Wunsch einer raschen Unterzeichnung des EVG- und des Generalvertrages.

120 29.04. Bundeskanzler Adenauer an den CDU/CSUFraktionsvorsitzenden Heinrich von Brentano

Adenauer nimmt zu der Aufforderung Stellung, mit der CDU/ CSU-Fraktion den Generalvertrag und die Zusatzverträge $z \mathrm{u}$ erörtern.

121 29.04. Bundeskanzler Adenauer an den Geschäftsführenden Vorsitzenden der Alliierten Hohen Kommission, Kirkpatrick

Adenauer entwickelt seine Vorstellungen zum Interzonenhandel. 
122

30.04. Aufzeichnung des Delegationsleiters Grewe

Grewe erörtert, ob der Truppenvertrag auch nach Inkrafttreten des EVG-Vertrages auf die französischen Streitkräfte in der Bundesrepublik angewendet werden darf.

123

02.05. Gespräch des Bundeskanzlers Adenauer mit dem Oberbefehlshaber der NATO-Streitkräfte in Europa, Eisenhower

Hauptthemen sind die europäische Integration und die Antwort der Drei Mächte auf die sowjetische Note vom 9. April 1952.

124

06.05. Aufzeichnung des Bundesministers Schäffer

Schäffer notiert den Verlauf eines Gesprächs mit dem Finanzberater im amerikanischen Hochkommissariat, Harris, über den finanziellen Verteidigungsbeitrag der Bundesrepublik.

06.05. Aufzeichnung des Rechtsberaters Kaufmann

Kaufmann erörtert Artikel 7 des Entwurfs vom 24. April 1952 für den Generalvertrag.

126

07.05. Generalkonsul I. Klasse Krekeler, Washington, an das Auswärtige Amt

Krekeler berichtet über Gespräche des Vortragenden Legationsrats Riesser, Washington, mit dem stellvertretenden britischen Botschafter in Washington, Steel, und dem Referenten im amerikanischen Außenministerium, Williams. Thema war die Antwort der Drei Mächte auf die sowjetische Note vom 9. April 1952.

127 08.05. Delegationsleiter Böhm, z.Z. Frankfurt/Main, an Ministerialdirektor Blankenhorn

Böhm gibt den kontroversen Verlauf eines Gesprächs mit Bundesminister Schäffer über die Wiedergutmachungsverhandlungen mit Israel und den jüdischen Organisationen wieder.

08.05. Aufzeichnung des Referenten von Plehwe

Plehwe erörtert die Kompetenzverteilung in der Bundesregierung bei der Zusammenarbeit mit internationalen Organisationen. von Falkenstein

Trützschler berichtet über ein Gespräch mit dem Mitarbeiter im amerikanischen Hochkommissariat, West. Gegenstand war der Beitritt der Bundesrepublik zum Weltpostverein. 
130 09.05. Aufzeichnung des Abteilungsleiters Mosler

Mosler erörtert die Anerkennung des Deutschen Roten Kreuzes durch das Internationale Rote Kreuz.

131 10.05. SPD-Vorsitzender Schumacher an Bundeskanzler Adenauer

Schumacher nimmt zu den Wiedergutmachungsverhandlungen mit Israel und den jüdischen Organisationen Stellung.

Böhm erörtert die unterschiedlichen Positionen, die im Bundeskabinett zu einem Angebot bei den Wiedergutmachungsverhandlungen mit Israel und den jüdischen Organisationen vertreten werden.

133 14.05. Generalkonsul I. Klasse Krekeler, Washington, an Bundeskanzler Adenauer

Krekeler weist auf die Bedeutung des EVG- und des Generalvertrages für die Außenpolitik der USA hin.

134 15.05. Aufzeichnung der Delegation für Auslandsschulden

Die Delegation unterbreitet einen Vorschlag zur Regelung der deutschen Auslandsschulden.

135 15.05. Aufzeichnung des Delegationsleiters Böhm

Böhm äußert sich zu einem Angebot der Bundesregierung bei den Wiedergutmachungsverhandlungen mit Israel und den jüdischen Organisation.

136 15.05. Aufzeichnung des Botschaftsrats a. D. Kordt

Ein Gespräch mit dem syrischen Generalkonsul Istuany über die Haltung der arabischen Staaten zu den israelischen Forderungen an die Bundesrepublik wird wiedergegeben. von Falkenstein

Trützschler erörtert die bevorstehende Tagung des Ministerkomitees des Europarats.

138 18.05. Delegationsleiter Böhm, z.Z. Frankfurt/Main, an Bundeskanzler Adenauer

Böhm bittet Adenauer, ihn vom Amt des Delegationsleiters bei den Wiedergutmachungsverhandlungen mit Israel und den jüdischen Organisationen zu entbinden. 
139 19.05. Aufzeichnung des Delegationsleiters Abs und des Ministerialdirektors Wolff, London

Abs und Wolff berichten von einem Gespräch mit dem Leiter der israelischen Delegation bei den Wiedergutmachungsverhandlungen, Shinnar, über die Leistungen der Bundesrepublik an Israel.

140 19.05. Außenministerkonferenz über eine Europäische Verteidigungsgemeinschaft in Paris

Themen sind unter anderem die Anwerbung von Staatsbürgern der Bundesrepublik zum Militärdienst für Nichtmitgliedstaaten, die Verwendung nationaler Streitkräfte durch die Mitgliedstaaten, der Sitz der EVG-Organe und die Sprachenregelung.

141 20.05. Außenministerkonferenz über eine Europäische Verteidigungsgemeinschaft in Paris

Im Mittelpunkt stehen die Geltungsdauer des EVG-Vertrages, Beschränkungen der Rüstungsproduktion der Bundesrepublik, die Verwendung nationaler Streitkräfte durch die Mitgliedstaaten sowie deren Verpflichtung, keine im Widerspruch zum EVGVertrag stehenden internationalen Verbindlichkeiten einzugehen.

142 20.05. Außenministerkonferenz über eine Europäische Verteidigungsgemeinschaft in Paris

Erörtert werden die Auswirkungen einer Wiedervereinigung Deutschlands auf die EVG.

143 21.05. Legationsrat I. Klasse Trützschler von Falkenstein an Oberst a. D. Graf von Kielmansegg, Paris

Trützschler weist Kielmansegg an, französischen Wünschen entgegenzuwirken, Teile des Truppenvertrages auch nach Inkrafttreten des EVG-Vertrages auf die französischen Streitkräfte in der Bundesrepublik anzuwenden.

144 21.05. Außenministerkonferenz über eine Europäische Verteidigungsgemeinschaft in Paris

Hauptthemen sind die Steuerbefreiung von EVG-Beamten, die Stimmengewichtung im Rat und die Verwendung nationaler Streitkräfte durch die Mitgliedstaaten.

145 23.05. CDU/CSU-Fraktionsvorsitzender Heinrich von Brentano an Bundeskanzler Adenauer

Brentano ersucht Adenauer, sich bei den Drei Mächten für eine Änderung des Artikels 7 Absatz 3 des Entwurfs für den Generalvertrag einzusetzen. 
146 24.05. Aufzeichnung des Delegationsleiters Böhm, z.Z. Frankfurt/Main

Böhm berichtet über Gespräche mit dem Vorsitzenden des World Jewish Congress, Goldmann, und der israelischen Delegation bei den Wiedergutmachungsverhandlungen. Adenauer

Schäfer erläutert die Haltung der FDP-Fraktion zu Artikel 7 Absatz 3 des Entwurfs für den Generalvertrag.

148 24./25. Außenministerkonferenz

05. Themen sind unter anderem der Zusammenhang zwischen EVGund Generalvertrag, Beschränkungen der Rüstungsproduktion der Bundesrepublik, Reparationen, die Anwendung des Truppenvertrages auf Streitkräfte von EVG-Mitgliedstaaten, Artikel 7 Absatz 3 des Generalvertrages und die Begnadigung von Deutschen, die wegen Kriegsverbrechen verurteilt wurden.

149 27.05. Generalkonsul I. Klasse Krekeler, Washington, an das Auswärtige Amt

Krekeler berichtet über ein Gespräch mit dem stellvertretenden amerikanischen Außenminister Bruce. Themen waren die Ratifizierung des Generalvertrages sowie die sowjetische Note vom 24. Mai 1952.

150 27.05. Aufzeichnung des Vortragenden Legationsrats Salat

Salat informiert über die Verhandlungen mit Frankreich über ein Kulturabkommen.

151 30.05. Runderlaß des Ministerialdirektors Blankenhorn

Blankenhorn unterrichtet die Auslandsvertretungen über die Unterzeichnung des EVG- und des Generalvertrages.

\section{07.06. Memorandum des Delegationsleiters Abs, London}

Abs äußert sich zu den Gesprächen des Delegationsleiters Böhm mit dem Vorsitzenden des World Jewish Congress, Goldmann, und israelischen Delegierten bei den Wiedergutmachungsverhandlungen am 23. Mai 1952 in Paris.

\section{13.06. Aufzeichnung des Referenten Frowein}

Frowein resümiert ein Gespräch, in dem Einvernehmen über ein Angebot der Bundesregierung für die Wiedergutmachungsverhandlungen mit Israel und den jüdischen Organisationen erzielt wurde. 
154 14.06. Aufzeichnung des Generalleutnants a.D. Speidel, Paris

Speidel leitet Informationen über eine mögliche französische Verzögerungstaktik bei der Ratifizierung des EVG-Vertrages weiter.

155 15.06. Aufzeichnung des Ministerialdirektors Blankenhorn

Blankenhorn faßt Gespräche zusammen, die er und Bundeskanzler Adenauer mit Vertretern der Drei Mächte über eine Antwort auf die sowjetische Note vom 24. Mai 1952 führten.

156 15.06. Aufzeichnung des Bundeskanzlers Adenauer

Adenauer formuliert Überlegungen zum Vorschlag einer VierMächte-Konferenz.

157 16.06. Aufzeichnung des Bundeskanzlers Adenauer

Adenauer lehnt die Einberufung einer Vier-Mächte-Konferenz ab.

158 17.06. Aufzeichnung des Legationsrats I. Klasse Brückner

Brückner berichtet über eine Besprechung des Handelspolitischen Ausschusses. Gegenstand war die Aufhebung der Zahlungssperre gegenüber der Volksrepublik China.

159 17.06. Gespräch des Bundeskanzlers Adenauer mit dem amerikanischen Hohen Kommissar McCloy

Themen sind die USA-Reise des amerikanischen Hohen Kommissars, die Ratifizierung des EVG- und des Generalvertrages, die Antwort auf die sowjetische Note vom 24. Mai 1952, die Präsidentschaftskandidatur des Generals Eisenhower und die Abberufung von McCloy.

18.06. Botschaftsrat von Walther, Paris, an das Auswärtige

Amt

Walther legt eine Einschätzung der Aussichten für die Ratifizierung des EVG-Vertrages in Frankreich vor.

161 19.06. Staatssekretär Hallstein an den Generalsekretär des Europarats, Paris

Hallstein erläutert die Gründe, aus denen die Erörterung des Memorandums der Bundesregierung vom 29. Februar 1952 über die Verletzung der Menschenrechte und Grundfreiheiten im Saargebiet nicht weiter vertagt werden könne. 
Im Mittelpunkt stehen die Wiedergutmachungsleistungen an Israel und die jüdischen Organisationen, die Ratifizierung des EVG- und des Generalvertrages sowie die Wiedervereinigung Deutschlands. Amt

Walther übermittelt und ergänzt Informationen des Gesandtschaftsrats Krapf zur Haltung der Drei Mächte hinsichtlich einer Vier-Mächte-Konferenz.

Blankenhorn gibt ein Gespräch mit dem amerikanischen Hohen Kommissar McCloy wieder, in dem die Antwort der Drei Mächte auf die sowjetische Note vom 24. Mai 1952 erörtert wurde.

Jansen berichtet über ein Gespräch mit dem Abteilungsleiter im luxemburgischen Außenministerium, Calmes. Thema war die Besetzung des Präsidentenamtes der Hohen Behörde der EGKS. amerikanischen Hohen Kommissar McCloy

Im Mittelpunkt stehen die Abschiedsreisen von McCloy durch Baden-Württemberg, Bayern und Hessen, die Antwort auf die sowjetische Note vom 24. Mai 1952 sowie die Ratifizierung des EVG- und Generalvertrages.

Hallstein resümiert Gespräche mit dem amerikanischen Außenminister Acheson in Berlin (West). Themen waren die Antwort auf die sowjetische Note vom 24. Mai 1952, die europäische Einigung, die Präsidentschaftswahlen in den USA, die Abberufung des Hohen Kommissars McCloy und die wegen Kriegsverbrechen von amerikanischen Militärgerichten verurteilten Deutschen.

Böker analysiert den Entwurf einer Antwort der Drei Mächte auf die sowjetische Note vom 24. Mai 1952. 
169 03.07. Aide-mémoire der Bundesregierung

Der AHK werden Änderungsvorschläge zum Entwurf einer Antwort der Drei Mächte auf die sowjetische Note vom 24. Mai 1952 unterbreitet.

170 07.07. Aufzeichnung des Staatssekretärs Hallstein

Hallstein nimmt Stellung zur Globalzahlung an Israel und die jüdischen Organisationen sowie zu individuellen Wiedergutmachungsleistungen an jüdische Opfer des Nationalsozialismus.

171

08.07. Delegationsleiter Abs, London, an Bundeskanzler Adenauer

S. 520

Abs äußert sich zu israelischen Vorschlägen für Sicherungsklauseln bei den Wiedergutmachungsverhandlungen.

172 10.07. Botschafter Du Mont, Den Haag, an das Auswärtige Amt

Du Mont berichtet über ein Gespräch mit dem niederländischen Kriegsminister Staf. Thema war die Stationierung von niederländischen Truppen in der Bundesrepublik.

173 15.07. Runderlaß des Staatssekretärs Hallstein

Hallstein kommentiert den Eden-Plan zur institutionellen Neugestaltung europäischer Organisationen.

174 16.07. Aufzeichnung des Staatssekretärs Hallstein

S. 530

Hallstein faßt ein Gespräch mit dem niederländischen Botschafter de Booy zusammen. Gegenstände waren die Unterredung des französischen Außenministers Schuman mit dem britischen Außenminister Eden über eine europäische politische Gemeinschaft und die Beteiligung Großbritanniens an der europäischen Einigung.

175 17.07. Aufzeichnung des Legationsrats I. Klasse Thierfelder

Thierfelder resümiert den Bericht des CDU-Abgeordneten Pünder über die Tagung des Ständigen Ausschusses der Beratenden Versammlung des Europarats vom 5. Juli 1952.

Thierfelder erörtert den Vorschlag des Ministerpräsidenten Hoffmann, Saarbrücken zum Sitz der EGKS-Behörden zu machen.

Adenauer skizziert Voraussetzungen und Form einer Europäisierung des Saargebiets. 
Blankenhorn vermerkt den Vorschlag des französischen Außenministers Schuman zur Europäisierung des Saargebiets, der im Verlauf der Außenministerkonferenz der EGKS-Staaten in Paris unterbreitet wurde.

Die Verhandlungen des Staatssekretärs Hallstein mit dem französischen Außenminister Schuman über eine Europäisierung des Saargebiets werden vorbereitet. Graf von Spreti

Blankenhorn äußert sich zu dem Vorschlag, die Stelle eines Parlamentarischen Staatssekretärs im Auswärtigen Amt einzurichten. Auswärtige Amt

Hardenberg berichtet über ein Gespräch mit Ministerpräsident Tito. Im Mittelpunkt stand die Freilassung der deutschen Kriegsgefangenen in Jugoslawien. Auswärtige Amt

Brentano faßt ein Gespräch mit Ministerpräsident de Gasperi zusammen. Themen waren die Saarfrage, ein Besuch des italienischen Ministerpräsidenten in Bonn und die Unterredung des Vorsitzenden der Sozialistischen Partei Italiens, Nenni, mit dem Generalsekretär des Zentralkomitees der KPdSU, Stalin, in Moskau. französischen Außenminister Schuman in Paris

Hallstein und Schuman erörtern Voraussetzungen und Modalitäten für eine Europäisierung des Saargebiets. französischen Außenminister Schuman

Adenauer nimmt Stellung zu den Gesprächen des Staatssekretärs Hallstein mit Schuman in Paris über die Saarfrage. französischen Außenminister Schuman in Paris

Erörtert werden die Verschiebung der Wahlen und die Zulassung der drei neuen Parteien im Saargebiet sowie ein Vorschlag von Schuman zur Lösung der Saarfrage. 
186 20.08. Aufzeichnung des Referenten Kaumann

Kaumann berichtet über ein Gespräch von Industrievertretern aus der Bundesrepublik mit Handelsvertretern der UdSSR in Kopenhagen.

187 20.08. Botschaftsrat Graf von Strachwitz, Rom, an das Auswärtige Amt

Strachwitz leitet Informationen des italienischen Staatssekretärs Taviani zum Besuch des Vorsitzenden der Sozialistischen Partei Italiens, Nenni, beim Generalsekretär des Zentralkomitees der KPdSU, Stalin, in Moskau weiter.

188 21.08. Aufzeichnung des Vortragenden Legationsrats von Etzdorf

Etzdorf faßt ein Gespräch mit dem Gesandten der Republik China (Taiwan), Tuan Mao-Lan, und dem Präsidenten der Taiwan Sugar Corporation, Yang, zusammen. Themen waren ein Waren- und Zahlungsabkommen sowie die Aufnahme diplomatischer Beziehungen.

189 22.08. Aufzeichnung des Referenten Biermann

Biermann nimmt zu einem Aufnahmeantrag der Bundesrepublik an die UNO Stellung.

190 25.08. Aufzeichnung des Legationsrats I. Klasse Thierfelder

Thierfelder erörtert Vorschläge zur Schaffung einer europäischen politischen Behörde.

191 25.08. Generalkonsul Hausenstein, Paris, an das Auswärtige Amt

Hausenstein berichtet über französische Reaktionen auf die sowjetische Note vom 23 . August 1952. Vorsitzenden der Alliierten Hohen Kommission, Donnelly

Hallstein erinnert an die Zusage der AHK vom 19. Dezember 1951, die Post- und Fernemldeüberwachung auf genau definierte Fälle zu beschränken.

Kaufmann legt ein Rechtsgutachten über die Tätigkeit des Untersuchungsausschusses Nr. 47 des Bundestages vor. 
194 29.08. Gespräch des Staatssekretärs Hallstein mit dem französischen Außenminister Schuman in Paris

Im Mittelpunkt stehen ein Vorschlag von Schuman und das Memorandum der Bundesregierung vom 18. August 1952 zur Lösung der Saarfrage.

195 03.09. Aufzeichnung des Referenten Biermann

Biermann schlägt vor, den Vortragenden Legationsrat Riesser zum Ständigen Beobachter der Bundesrepublik bei der UNO zu ernennen. von Falkenstein

Trützschler bittet um Weisung in der Frage, ob die Drei Mächte den Abschlußbericht der UNO-Kommission für gesamtdeutsche Wahlen auf die Tagesordnung der UNO-Generalversammlung setzen lassen sollen.

Etzdorf nimmt zu den arabischen Argumenten gegen die Wiedergutmachungsverhandlungen mit Israel Stellung. Ministerialdirektor Blankenhorn

Krekeler berichtet über ein Gespräch mit dem amerikanischen Außenminister Acheson. Themen waren die Ratifizierung des EVG- und des Generalvertrages, die wegen Kriegsverbrechen verurteilten Deutschen, die Saarfrage und die Unterzeichnung des Abkommens mit Israel.

199 09.09. Aufzeichnung des Vortragenden Legationsrats von Kessel, Paris

Kessel berichtet von einem Gespräch mit dem belgischen Gesandten de Staerke über den Vorschlag des französischen AuBenministers Schuman, die Saar zu europäisieren.

Adenauer faßt ein Gespräch mit dem französischen Außenminister Schuman über die Saarfrage zusammen.

Etzdorf resümiert ein Gespräch des Staatssekretärs Hallstein mit dem Vorsitzenden des Verbandes Deutscher Landsmannschaften, Lodgman von Auen, über die Zusammenarbeit des Verbandes mit dem Auswärtigen Amt. 
Pfeiffer empfiehlt eine Regierungserklärung zum Abschlußbericht des Untersuchungsausschusses Nr. 47 des Bundestages.

Amt

Nöldeke berichtet von einem Gespräch mit dem Staatssekretär im dänischen Außenministerium, Svenningsen, über die Aufnahme von Verhandlungen über ein Minderheitenabkommen.

16.09. Aufzeichnung des Vortragenden Legationsrats von Etzdorf

Etzdorf rät von einer Good-will-Mission in die arabischen Staaten $a b$ und schlägt statt dessen die baldige Entsendung der vorgesehenen diplomatischen Vertreter vor. Parteivorsitzenden im Saargebiet

Adenauer schildert die Entwicklung der Verhandlungen mit Frankreich über die Saarfrage und erörtert mit den Parteivorsitzenden die strittigen Fragen.

Grewe erläutert seine Auslegung des Artikels 7 Absatz 3 des Generalvertrages.

Adenauer ersucht Brentano, sich für eine baldige Ratifizierung des EVG- und des Generalvertrages einzusetzen.

Themen sind die Haltung Italiens zur europäischen Einigung, die italienische Innenpolitik, die Triest-Frage sowie die wirtschaftlichen und kulturellen Beziehungen.

Blankenhorn informiert über den Inhalt des Abkommens mit Israel sowie die Reaktionen in Israel und den arabischen Staaten. mit dem französischen Delegationsleiter Alphand in Paris

Thematisiert werden die Rechtsstellung und Dislozierung der französischen Truppen in der Bundesrepublik. 
Etzdorf erörtert arabische Reaktionen auf die Unterzeichnung des Abkommens mit Israel. Außenminister Schuman

Adenauer schlägt Schuman einen auf fünf Jahre befristeten Modus vivendi in der Saarfrage vor.

Hallstein berichtet von einem Gespräch mit dem französischen Außenminister Schuman über das Schreiben des Bundeskanzlers Adenauer vom Vortag.

214 06.10. Aufzeichnung des Referenten Born

Eine Ressortbesprechung über den Beitritt der Bundesrepublik zu den Genfer Rotkreuz-Abkommen wird wiedergegeben.

Grewe erörtert die im Ausschuß des Bundestages für das Besatzungsstatut und auswärtige Angelegenheiten vorgebrachte Auslegung des Artikels 7 Absatz 3 des Generalvertrages.

Pawelke berichtet über ein Gespräch mit dem ägyptischen Außenminister Tajeh. Themen waren die Entsendung einer arabischen Delegation und das Abkommen mit Israel.

16.10. Bundeskanzler Adenauer an den französischen Außenminister Schuman

Adenauer konkretisiert seine Vorschläge zur Lösung der Saarfrage. Wahlendorf

Schwarz-Liebermann gibt eine Sitzung der stellvertretenden AuBenminister der EGKS-Staaten wieder. Gegenstand war der Fragebogen zur Gründung einer europäischen politischen Gemeinschaft für die Ad-hoc-Versammlung.

Hartlieb berichtet über ein Gespräch mit dem französischen Ministerbeauftragten im Europarat, Jouve. Themen waren die Saarfrage, die Europarat-Konventionen, der Eden-Plan und die wirtschaftliche Erschließung der überseeischen Gebiete der Mitgliedstaaten des Europarats. 

mit dem Exekutivsekretär der ECE, Myrdal

Erörtert wird die Mitarbeit der Bundesrepublik in der ECE.

Adenauer legt seine Auffassung über die Vertretung der Bundesrepublik im Ministerrat der EGKS dar. mit einer arabischen Delegation

Die Forderungen der arabischen Delegation im Zusammenhang mit dem Abkommen mit Israel werden erörtert.

Amt

Gegenstand eines abendlichen Gesprächs in der Botschaft in Paris auf Einladung des Vorsitzenden des Verfassungsausschusses der Ad-hoc-Versammlung, Heinrich von Brentano, ist die Errichtung einer europäischen politischen Gemeinschaft.

Pawelke berichtet von einem Gespräch mit Ministerpräsident Naguib über die Demarche der arabischen Delegation in der Bundesrepublik.

Blankenhorn resümiert eine Aussprache zwischen Staatssekretär Hallstein und dem amerikanischen Botschafter Kennan über die Saarfrage.

Böker erörtert die Folgen der Ratifizierung des Abkommens mit Israel im Hinblick auf den Konflikt mit der Arabischen Liga und schlägt eine Einschaltung der UNO vor.

Pawelke gibt ein Gespräch mit dem Staatssekretär im ägyptischen Außenministerium, Hakki Pasha, über die Haltung der Arabischen Liga zum Abkommen mit Israel wieder.

228

08.11. Vortragender Legationsrat Riesser, New York (UNO), an Staatssekretär Hallstein

Riesser erörtert das Verfahren und die Erfolgsaussichten, den Konflikt mit der Arabischen Liga durch Einschaltung der UNO beizulegen. 

Auswärtige Amt

Bayern berichtet über ein Gespräch mit Staatschef Franco anläßlich der Übergabe des Beglaubigungsschreibens. Themen waren die deutsch-spanischen Beziehungen vor 1945 und die bilateralen Wirtschaftsbeziehungen. Gasperi

Adenauer bittet de Gasperi, sich für eine baldige Ratifizierung des EVG-Vertrages einzusetzen.

231 17.11. Aufzeichnung des Staatssekretärs Hallstein

Hallstein resümiert ein Gespräch mit dem französischen Verteidigungsminister Pleven. Im Mittelpunkt standen die Ratifizierung des EVG-Vertrages, die Saarfrage und die bilaterale wirtschaftliche Zusammenarbeit.

Vialon bilanziert die Verhandlungen im Interimsausschuß der EVG-Konferenz.

Ophüls schlägt die Einrichtung föderaler europäischer Organe zur Koordinierung der Außenpolitik vor.

Sigrist faßt eine Hausbesprechung zusammen, deren Gegenstand die Beilegung des Konflikts mit der Arabischen Liga war.

Schlegelberger resümiert die Verhandlungen über Kulturabkommen mit den USA, Italien, Frankreich, Spanien und Großbritannien.

In Vorbereitung eines Gesprächs mit dem Vorsitzenden des World Jewish Congress, Goldmann, faßt Pauls Vorschläge für eine Beilegung des Konflikts mit der Arabischen Liga zusammen. 
Krekeler erörtert mutmaßliche amerikanische Reaktionen auf ein Scheitern der Ratifizierung des EVG- und des Generalvertrages.

Blankenhorn berichtet von einem Gespräch mit dem Vorsitzenden des World Jewish Congress, Goldmann. Thema war die Beilegung des Konflikts mit der Arabischen Liga durch Einschaltung der UNO.

240 01.12. Aufzeichnung des Ministerialdirektors Blankenhorn

Blankenhorn resümiert ein Gespräch des Bundeskanzlers Adenauer mit dem indonesischen Außenminister Mukarto Notowidigdo über die wirtschaftlichen und militärischen Beziehungen.

01.12. Bundeskanzler Adenauer an den ehemaligen amerikanischen Hohen Kommissar McCloy

Adenauer schlägt die Ernennung eines Ständigen amerikanischen Botschafters für Europa vor. Auswärtige Amt

Kessel berichtet über ein Gespräch mit dem Vorsitzenden der EVG-Konferenz, Alphand, über die noch zu behandelnden Themen. Bundeskanzler Adenauer

Krekeler informiert über ein Gespräch mit dem amerikanischen Außenminister Acheson. Thema war die Ratifizierung des EVG- und des Generalvertrages durch den Bundestag.

Böker gibt ein Gespräch des Staatssekretärs Hallstein mit dem Vertreter der Jewish Agency for Palestine, Josephthal, und dem Abteilungsleiter im israelischen Außenministerium, Jachil, wieder. Im Mittelpunkt stand das Abkommen mit Israel. 
246 11.12. Vortragender Legationsrat von Kessel, Paris, an das Auswärtige Amt

Kessel berichtet von einem Gespräch mit dem Vorsitzenden der EVG-Konferenz, Alphand. Thema war die Einsetzung einer ständigen Konferenz der Regierungschefs.

247 11.12. Aufzeichnung des Referenten Schwarz-Liebermann von Wahlendorf

Schwarz-Liebermann legt die Fortschritte bei den Verhandlungen über das Statut einer europäischen politischen Gemeinschaft dar.

248 12.12. Aufzeichnung des Staatssekretärs Hallstein

Hallstein instruiert Botschafter Pawelke für ein Gespräch mit Ministerpräsident Naguib zur Beilegung des Konflikts mit der Arabischen Liga.

249 20.12. Aufzeichnung des Botschaftsrats a. D. Kordt

Kordt macht Vorschläge für die Grenzverhandlungen mit Belgien.

250 21.12. Aufzeichnung des Staatssekretärs Hallstein

Hallstein gibt einen Überblick über den Aufbau des Auswärtigen Amts und des Auswärtigen Dienstes.

251 27.12. Aufzeichnung des Legationsrats I. Klasse Böker

Böker resümiert ein Gespräch mit dem stellvertretenden amerikanischen Hohen Kommissar, Reber, über eine Erklärung von General Eisenhower zur Ratifizierung des EVG-Vertrages.

29.12. Staatssekretär Hallstein an Botschafter Pawelke, Kairo

Hallstein instruiert Pawelke für ein Gespräch mit Ministerpräsident Naguib zur Beilegung des Konflikts mit der Arabischen Liga.

253 30.12. Botschafter Pawelke, Kairo, an das Auswärtige Amt

Pawelke berichtet über bevorstehende Wirtschaftsverhandlungen zwischen Ägypten und der DDR. 


\section{Literaturverzeichnis}

AAPD

AAPD, Hohe Kommissare 1949-1951

AAPD, Hohe Kommissare 1952

ABS, Entscheidungen

ACTES

ADAP, D, II

ADAP, D, XI/1

ADENAUER, Briefe 1949-1951

ADENAUER, Briefe 1951-1953

ADENAUER, Erinnerungen 1945-1953

ADENAUER, Erinnerungen 1953-1955

AdG
Akten zur Auswärtigen Politik der Bundesrepublik Deutschland, hrsg. im Auftrag des Auswärtigen Amts vom Institut für Zeitgeschichte. Jahresband 1949/50. Jahresband 1951, München 1997 und 1999.

Akten zur Auswärtigen Politik der Bundesrepublik Deutschland. Band 1: Adenauer und die Hohen Kommissare 1949-1951, hrsg. von Hans-Peter Schwarz in Verbindung mit Reiner Pommerin, München 1989.

Akten zur Auswärtigen Politik der Bundesrepublik Deutschland. Band 2: Adenauer und die Hohen Kommissare 1952, hrsg. von Hans-Peter Schwarz in Verbindung mit Reiner Pommerin, München 1990.

Hermann J. Abs, Entscheidungen 1949-1953. Die Entstehung des Londoner Schuldenabkommens, 2. Auflage, Mainz 1991.

Les Actes de l'Union postale universelle révisés à Paris 1947 et annotés par les soins du Bureau international, hrsg. vom Bureau international de l'Union postale universelle, Bern 1949.

Akten zur deutschen auswärtigen Politik 1918 1945. Serie D (1937-1945). Band II: Deutschland und die Tschechoslowakei (Oktober 1937September 1938), Baden-Baden 1950.

Akten zur deutschen auswärtigen Politik 1918 1945. Serie D (1937-1945). Band XI, 1. Halbband: Die Kriegsjahre (1. September-13. November 1940), Bonn 1964.

Adenauer. Rhöndorfer Ausgabe: Briefe 19491951, hrsg. von Rudolf Morsey und Hans-Peter Schwarz, bearbeitet von Hans Peter Mensing, Berlin 1985.

Adenauer. Rhöndorfer Ausgabe: Briefe 19511953, hrsg. von Rudolf Morsey und Hans-Peter Schwarz, bearbeitet von Hans Peter Mensing, Berlin 1987.

Konrad Adenauer, Erinnerungen 1945-1953, Stuttgart 1965.

Konrad Adenauer, Erinnerungen 1953-1955, Stuttgart 1966.

Keesing's Archiv der Gegenwart, zusammengestellt von Heinrich von Siegler, Wien 1950 ff. 


\author{
AMTSBLATT DER AHK \\ AMTSBLATT DER MILITÄRREGIERUNG \\ DEUTSCHLAND (Britisches Kontroll- \\ gebiet)
}

AMTSBLATT DES KONTROLLRATS

AMTSBLATT DES SAARLANDES

AUSSENPOLITIK DER DDR I

AUSWÄRTIGER AUSSCHUSS

BDFD I

BDFD II

BEMUUHUNGEN

BERICHT

BILLOTTE, Kritik
Amtsblatt der Alliierten Hohen Kommission in Deutschland. Nr. 1-72 [o.O.] 1949-1951.

Amtsblatt der Militärregierung Deutschland. Nr. 1-3: Kontrollgebiet der 21. Armeegruppe; Nr. 4-28: Britisches Kontrollgebiet; Nr. 29-36: Amtsblatt der Kontrollkommission-Deutschland (Britische Zone), [1945-1949].

Amtsblatt des Kontrollrats in Deutschland, hrsg. vom Alliierten Sekretariat. Nr. 1-19, Berlin $1945-1948$.

Amtsblatt des Saarlandes 1947-1952, hrsg. vom Informationsamt der Regierung des Saarlandes, Saarbrücken 1947-1952.

Dokumente zur Außenpolitik der Regierung der Deutschen Demokratischen Republik. Band I: Von der Gründung der Deutschen Demokratischen Republik am 7. Oktober 1949 bis zur Souveränitätserklärung am 25. März 1954, hrsg. vom Deutschen Institut für Zeitgeschichte, Berlin [Ost] 1954.

Der Auswärtige Ausschuß des Deutschen Bundestages. Sitzungsprotokolle 1949-1953. 1. Halbband: Oktober 1949 bis Mai 1952; 2. Halbband: Juni 1952 bis August 1953 und Unterausschuß „Besatzungsregime ${ }^{\text {“ }} 1951 / 52$, bearbeitet von Wolfgang Hölscher, Düsseldorf 1998.

Die Bundesrepublik Deutschland und Frankreich. Dokumente 1949 bis 1963. Band 1: Außenpolitik und Diplomatie, hrsg. von Horst Möller und Klaus Hildebrand, bearbeitet von Ulrich Lappenküper, München 1997.

Die Bundesrepublik Deutschland und Frankreich: Dokumente 1949 bis 1963. Band 2: Wirtschaft, hrsg. von Horst Möller und Klaus Hildebrand, bearbeitet von Andreas Wilkens, München 1997.

Die Bemühungen der Bundesrepublik um Wiederherstellung der Einheit Deutschlands durch gesamtdeutsche Wahlen. Dokumente und Akten. I. Teil: Oktober 1949-Oktober 1953, hrsg. vom Bundesministerium für gesamtdeutsche Fragen, 4. erweiterte Auflage, Bonn 1958.

Bericht der Konferenz über Deutsche Auslandsschulden, London 1952.

Pierre Billotte, Noch ist es Zeit! Kritik und Vorschläge zur Verteidigung des Westens, BadenBaden 1951. 
BöHM, Abkommen

BR DRUCKSACHEN

BR SITZUNGSBERICHTE

BRIEFWECHSEL ADENAUER-BRENTANO

BT ANLAGEN

BT STENOGRAPHISCHE BERICHTE

SENAT UND BÜRGERSCHAFT

BULLETIN

BUNDESANZEIGER

BUNDESGESETZBLATT

CDU/CSU-FRAKTION

CHARTER OF THE UNITED NATIONS

Council of Europe, Consultative ASSEMBLY, FOURTH SESSION, TEXTS

COUNCIL OF EuRoPe, Consultative ASSEMBLY, DEBATES, 1952
Franz Böhm, Das deutsch-israelische Abkommen 1952, in: Konrad Adenauer und seine Zeit. Politik und Persönlichkeit des ersten Bundeskanzlers. Beiträge von Weg- und Zeitgenossen, hrsg. von Dieter Blumenwitz, Klaus Gotto, Hans Maier, Konrad Repgen und Hans-Peter Schwarz, Stuttgart 1976.

Deutscher Bundesrat. Drucksachen, Bonn $1949 \mathrm{ff}$.

Deutscher Bundesrat. Sitzungsberichte, Bonn $1949 \mathrm{ff}$.

Arnulf Baring, Sehr verehrter Herr Bundeskanzler! Heinrich von Brentano im Briefwechsel mit Konrad Adenauer 1949-1964, Hamburg 1974.

Verhandlungen des Deutschen Bundestages. Anlagen zu den Stenographischen Berichten, Bonn $1949 \mathrm{ff}$.

Verhandlungen des Deutschen Bundestages. Stenographische Berichte, Bonn 1949 ff.

Verhandlungen zwischen dem Senate und der Bürgerschaft 1952, Bremen [o.J.].

Bulletin des Presse- und Informationsamtes der Bundesregierung, Bonn $1952 \mathrm{ff}$.

Bundesanzeiger (bis August 1950: Bundesanzeiger und Öffentlicher Anzeiger für das Vereinigte Wirtschaftsgebiet. Bekanntmachungsorgan für das französische Besatzungsgebiet. Verkündungsorgan für die Verlautbarungen des Einfuhrausschusses), hrsg. vom Bundesminister der Justiz, Bonn 1949 ff.

Bundesgesetzblatt, hrsg. vom Bundesminister der Justiz, Köln 1949 ff.

Die CDU/CSU-Fraktion im Deutschen Bundestag 1949-1966. 1. Halbband: Sitzungsprotokolle 1949-1953, bearbeitet von Helge Heidemeyer, Düsseldorf 1998.

Charter of the United Nations. Commentary and Documents, hrsg. von Leland M. Goodrich, Edvard Hambro und Anne Patricia Simons, 3. überarbeitete Auflage, New York/London 1969.

Council of Europe. Consultative Assembly. Fourth Ordinary Session. Texts Adopted by the Assembly, Straßburg 1952.

Council of Europe. Consultative Assembly. Fourth Ordinary Session. Official Report of Debates. 5 Bände, Straßburg 1952. 
COUNCIL OF EUROPE, MINISTERS, DOCUMENTS, 1952

CounCIL OF EUROPE, Ministers, RESOLUTIONS, 1949-1951

COUNCIL OF EUROPE, MINISTERS, RESOLUTIONS, 1952

DBPO IL/1

DEPARTMENT OF STATE BULLETIN

DEUTSCHE AUSLANDSSCHULDEN

DEUTSCHES VERMÖGEN IM AUSLAND

DEUTSCH-ISRAELISCHER DiALOG

DEUTSCHLANDS WEG NACH ISRAEL

DIPLOMATISCHER KURIER

DOCUMENTS 1951

DOCUMENTS 1952
Council of Europe. Documents of the Committee of Ministers, Straßburg 1952.

Council of Europe. Resolutions of the Committee of Ministers. First to the Ninth Session 1949-1951, Straßburg 1952.

Council of Europe. Resolutions (1952) of the Committee of Ministers, Straßburg 1953.

Documents on British Policy Overseas. Series II. Band 1: The Schuman Plan, the Council of Europe and Western European Integration, May 1950-December 1952, hrsg. von Roger Bullen und M. E. Pelly unter Mitarbeit von H. J. Yasamee und G. Bennett, London 1986.

The Department of State Bulletin. The Official Weekly Record of United States Foreign Policy, Washington D.C. 1939 ff.

Deutsche Auslandsschulden. Dokumente zu den internationalen Verhandlungen, Oktober 1950 bis Juli 1951, hrsg. vom Auswärtigen Amt, dem Bundesministerium für Wirtschaft und dem Bundesministerium für den Marshallplan, Hameln [1951].

Deutsches Vermögen im Ausland. Internationale Vereinbarungen und ausländische Gesetzgebung. Mit Unterstützung des Bundesministeriums der Finanzen, des Bundesministeriums für Wirtschaft, des Bundesministeriums für den Marshallplan und der Bank deutscher Länder hrsg. vom Bundesministerium der Justiz, bearbeitet von Otto Böhmer, Konrad Duden und Hermann Jansen, Köln 1951.

Der deutsch-israelische Dialog. Dokumentation eines erregenden Kapitels deutscher Außenpolitik, hrsg. von Rolf Vogel, Teil I: Politik. Band 1, München/New York/London/Paris 1987.

Deutschlands Weg nach Israel. Eine Dokumentation mit einem Geleitwort von Konrad Adenauer, hrsg. von Rolf Vogel, Stuttgart 1967.

Diplomatischer Kurier in Verbindung mit Diplomatische Korrespondenz, Köln 1953.

Documents on the Foreign Policy of Israel. Band 6: 1951, hrsg. von Yemima Rosenthal, Jerusalem 1991.

Documents on the Foreign Policy of Israel. Band 7: 1952, hrsg. von Yehoshua Freundlich, Jerusalem 1992. 


\section{DOCUMENTS. COMPANION VOLUME 1952}

\author{
DOKUMENTE DES GETEILTEN \\ DEUTSCHLAND
}

DOKUMENTE ZUR BERLIN-FraGE

\section{DOKUMENTE ZUR SUDETENDEUTSCHEN FRAGE}

DzD I/2

DZD II/1

DzD II/2

ECKARDT, Leben

ECOSOC 1947

ECOSOC 1952
Documents on the Foreign Policy of Israel. Band 7: 1952 Companion Volume, hrsg. von Yehoshua Freundlich, Jerusalem 1992.

Dokumente des geteilten Deutschland. Quellentexte zur Rechtslage des Deutschen Reiches, der Bundesrepublik Deutschland und der Deutschen Demokratischen Republik. Band 1, hrsg. von Ingo von Münch, 2. Auflage, Stuttgart 1976.

Dokumente zur Berlin-Frage 1944-1966, hrsg. vom Forschungsinstitut der Deutschen Gesellschaft für Auswärtige Politik e.V., Bonn in Zusammenarbeit mit dem Senat von Berlin, 3. durchgesehene und erweiterte Auflage, München 1967.

Dokumente zur Sudetendeutschen Frage 19161967, hrsg. von Ernst Nittner, überarbeitete und ergänzte Neuauflage der „Dokumente zur Sudetendeutschen Frage 1918-1959“, München 1967.

Dokumente zur Deutschlandpolitik. I. Reihe: Vom 3. September 1939 bis 8. Mai 1945. Band 2: Amerikanische Deutschlandpolitik 11. August 1941 bis 31. Dezember 1942, hrsg. vom Bundesministerium für Innerdeutsche Beziehungen, bearbeitet von Marie-Luise Goldbach, Frankfurt am Main 1986.

Dokumente zur Deutschlandpolitik. II. Reihe: Vom 9. Mai 1945 bis 4. Mai 1955. Band 1: Die Konferenz von Potsdam, 3 Teilbände, hrsg. vom Bundesminister des Innern, bearbeitet von Gisela Biewer, Neuwied/Frankfurt am Main 1992.

Dokumente zur Deutschlandpolitik. II. Reihe: Vom 9. Mai 1945 bis 4. Mai 1955. Band 2: Die Konstituierung der Bundesrepublik Deutschland und der Deutschen Demokratischen Republik 7. September bis 31. Dezember 1949, hrsg. vom Bundesministerium des Innern unter Mitwirkung des Bundesarchivs, bearbeitet von Hanns Jürgen Küsters unter Mitarbeit von Daniel Hoffmann, München 1996.

Felix von Eckardt, Ein unordentliches Leben. Lebenserinnerungen, Düsseldorf/Wien 1967.

United Nations. Economic and Social Council. Official Records. Second Year: Fourth Session. From the fifty-first meeting (28 February 1947) to the eighty-fourth meeting (29 March 1947), New York 1947.

United Nations. Economic and Social Council. Official Records. Fourteenth Session. 20 May1 August 1952, New York 1952. 
ENTSCHEIDUNGEN

EUROPA-ARCHIV

FRUS 1947

FRUS 1948

FRUS 1949

FRUS 1951

FRUS 1952-1954

GERMANY 1947-1949

GESAMTDEUTSCHER AUSSCHUSS

GESETZBLATT

GESETZBLATT DER DDR

GESETZ- UND VERORDNUNGSBLATT

GoldMANN, Staatsmann

HAAS, Beitrag
Entscheidungen des Bundesverfassungsgerichts, Band 2, hrsg. von den Mitgliedern des Bundesverfassungsgerichts, Tübingen 1953.

Europa-Archiv. Zeitschrift für Internationale Politik, Frankfurt am Main 1946 ff.

Foreign Relations of the United States, 1947. Band II: Council of Foreign Ministers; Germany and Austria, Washington D.C. 1972.

Foreign Relations of the United States, 1948. Band II: Germany and Austria, Washington D.C. 1973.

Foreign Relations of the United States, 1949. Band III: Council of Foreign Ministers; Germany and Austria, Washington D.C. 1974.

Foreign Relations of the United States, 1951. Band I: National Security Affairs; Foreign Economic Policy; Band III: European Security and the German Question, 2 Teilbände, Washington D.C. 1979-1981.

Foreign Relations of the United States, 19521954. Band V: Western European Security, 2 Teilbände; Band VI: Western Europe and Canada, 2 Teilbände; Band VII: Germany and Austria, 2 Teilbände; Band IX: The Near and Middle East, 2 Teilbände, Washington D.C. 1983-1986.

Germany 1947-1949. The Story in Documents, hrsg. vom Department of State, Washington D.C. 1950 .

Der Gesamtdeutsche Ausschuß. Sitzungsprotokolle des Ausschusses für gesamtdeutsche Fragen des Deutschen Bundestages 1949-1953, bearbeitet von Andreas Biefang, Düsseldorf 1998.

Gesetzblatt der Verwaltung des Vereinigten Wirtschaftsgebietes, Frankfurt am Main 1949.

Gesetzblatt der Deutschen Demokratischen Republik, Berlin [Ost] 1949 ff.

Gesetz- und Verordnungsblatt des Wirtschaftsrates des Vereinigten Wirtschaftsgebietes, Frankfurt am Main 1948.

Nahum Goldmann, Staatsmann ohne Staat. Autobiographie, Köln/Berlin 1970.

Wilhelm Haas, Beitrag zur Geschichte der Entstehung des Auswärtigen Dienstes der Bundesrepublik Deutschland, Bonn 1969. 
JOURNAL OFFICIEL, CONSEIL DE LA
REPUBLIQUE

KABINETTSPROTOKOLLE

LEAGUE OF ARAB STATES

LENZ, Zentrum

MINISTERIALBLATT

MinisTERIALBLATT RHEINLAND-PFALZ

PUBLIC PAPERS, EISENHOWER 1953

PUBlic PAPERS, TRUMAN 1952-53

RAPPORTS

REICHSGESETZBLATT

SAARPOLITIK
Journal Officiel de la République Française. Débats Parlementaires. Conseil de la République. Compte rendue in extenso des séances. Questions écrites et réponses des Ministres à ces questions, Paris $1949 \mathrm{ff}$.

Die Kabinettsprotokolle der Bundesregierung, hrsg. für das Bundesarchiv von Hans Booms. Band 2: 1950, bearbeitet von Ulrich Enders und Konrad Reiser; Band 5: 1952, bearbeitet von Kai von Jena; Band 6: 1953, bearbeitet von Ulrich Enders und Konrad Reiser, Boppard am Rhein 1984-1989.

Hussein A. Hassouna, The League of Arab States and Regional Disputes. A Study of Middle East Conflicts, New York/Leiden 1975.

Im Zentrum der Macht. Das Tagebuch von Staatssekretär Lenz 1951-1953, bearbeitet von Klaus Gotto, Hans-Otto Kleinmann und Reinhard Schreiner, Düsseldorf 1989.

Gemeinsames Ministerialblatt des Bundesministers des Innern, des Bundesministers für Vertriebene, des Bundesministers für Wohnungsbau, des Bundesministers für gesamtdeutsche Fragen und des Bundesministers für Angelegenheiten des Bundesrates, hrsg. vom Bundesministerium des Innern, Berlin/Köln 1951.

Ministerialblatt der Landesregierung von Rheinland-Pfalz, hrsg. von der Staatskanzlei der Landesregierung Rheinland-Pfalz, Neuwied $1949 \mathrm{ff}$.

Public Papers of the Presidents of the United States. Dwight D. Eisenhower. Containing the Public Messages, Speeches and Statements of the President, January 1 to December 31, 1953, Washington D. C. 1960.

Public Papers of the Presidents of the United States. Harry S. Truman. Containing the Public Messages, Speeches, and Statements of the President, January 1, 1952 to January 20, 1953, Washington D. C. 1966.

Les Rapports Mensuels d'André François-Poncet, Haut-Commissaire français en Allemagne 1949-1955, bearbeitet von Hans Manfred Bock, 2 Bände, Paris 1996.

Reichsgesetzblatt, hrsg. vom Reichsministerium des Innern, Berlin 1919-1945.

Robert H. Schmidt, Saarpolitik 1945-1957. Band 2: Entfaltung der Saarpolitik zwischen "Wirtschaftsanschlu $\beta^{\prime \prime}$ und "Europäisierung 1945-1953, Berlin 1960. 
SCHLESWIGFRAGE

SCHUMACHER, Reden

SHINNAR, Bericht

UN GENERAL ASSEMBLY, AD HOC POLITICAL COMMITTEE, 1951-52

\section{UN GENERAL ASSEMBLY, SEVENTH SESSION, ANNEXES}

UN General ASSEMBLy, SEVENTH SESSion, Plenary MEETings

UNITED NATIONS RESOLUTIONS I

UNITED STATES. STATUTES AT LARGE 1946

UNITED STATES. STATUTES AT LARGE 1951

UNTS

VERFASSUNGSAUSSCHUSS

VERHANDLUNGEN
Die Schleswig-Frage seit 1945. Dokumente zur Rechtsstellung der Minderheiten beiderseits der deutsch-dänischen Grenze, hrsg. von Eberhard Jäckel, Frankfurt am Main/Berlin 1959.

Kurt Schumacher, Reden-Schriften-Korrespondenzen 1945-1952, hrsg. von Willy Albrecht, Berlin/Bonn 1985.

Felix E. Shinnar, Bericht eines Beauftragten. Die Deutsch-Israelischen Beziehungen 19511966, Tübingen 1967.

United Nations. Official Records of the General Assembly. Sixth Session. Ad Hoc Political Committee, Summary Records of Meetings, 19 November 1951 to 31 January 1952, Paris [o. J.].

United Nations. Official Records of the General Assembly. Seventh Session. Annexes Volume II. From 14 October 1952 to 28 August 1953, New York 1954.

United Nations. Official Records of the General Assembly, Seventh Session. Plenary Meetings, Verbatim Records of Meetings 14 October 1952 to 28 August 1953, New York 1953.

United Nations Resolutions. Series I: Resolutions Adopted by the General Assembly, hrsg. von Dusan J. Djonovich, New York 1972 ff.

United States. Statutes at Large Containing the Laws and Concurrent Resolutions Enacted during the Second Session of the Seventy-Ninth Congress of the United States of America 1946. Band 60 in 2 Teilbänden, Washington D.C. 1947.

United States. Statutes at Large Containing the Laws and Concurrent Resolutions Enacted during the First Session of the Eighty-Second Congress of the United States of America 1951 and Reorganization Plan, Amendment to the Constitution, and Proclamations. Band 65, Washington D.C. 1952.

United Nations Treaty Series. Treaties and International Agreements Registered or Filed and Recorded with the Secretariat of the United Nations, [New York] 1946 ff.

Leitfaden und amtliche Dokumente des Verfassungsausschusses, hrsg. vom Sekretariat des Verfassungsausschusses, Paris 1953.

Verhandlungen der Bremischen Bürgerschaft, Bremen 1952. 
VOGEL, Diplomat

WEHRBEITRAG I

WEHRBEITRAG II

YEARBOOK OF THE UNITED NATIONS

ZOLLANSCHLUSS I
Georg Vogel, Diplomat unter Hitler und Adenauer, Düsseldorf/Wien 1969.

Der Kampf um den Wehrbeitrag. 1. Halbband: Die Feststellungsklage, hrsg. vom Institut für Staatslehre und Politik e. V., München 1952.

Der Kampf um den Wehrbeitrag. 2. Halbband: Das Gutachtenverfahren (30.7.-15.12.1952), hrsg. vom Institut für Staatslehre und Politik e. V., München 1953.

Yearbook of the United Nations 1950 und 1952, hrsg. vom Department of Public Information United Nations, New York 1951 und 1953.

Albert Calmes, Der Zollanschluss des Grossherzogtums Luxemburg an Deutschland (18421918). Band I: Der Eintritt Luxemburgs in den deutschen Zollverein (1839-1842), Luxemburg 1919. 


\section{Abkürzungsverzeichnis}

\begin{tabular}{|c|c|c|c|}
\hline AA & Auswärtiges Amt & DPS & Demokratische Partei Saar \\
\hline a.D. & außer Dienst & DRK & Deutsches Rotes Kreuz \\
\hline AG & Aktiengesellschaft & DSP & Deutsche Sozialdemokra- \\
\hline AGSEC & Allied General Secretariat & & \\
\hline AHK & Alliierte Hohe Kommission & DVP & Demokratische Volkspartei \\
\hline $\mathrm{AR}$ & Amtsrat & ECA & $\begin{array}{l}\text { Economic Cooperation } \\
\text { Administration }\end{array}$ \\
\hline $\begin{array}{l}\text { Ber. Nr. } \\
\text { BGBl. }\end{array}$ & $\begin{array}{l}\text { Bericht-Nummer } \\
\text { Bundesgesetzblatt }\end{array}$ & ECE & $\begin{array}{l}\text { Economic Commission for } \\
\text { Europe }\end{array}$ \\
\hline BHE & $\begin{array}{l}\text { Bund der Heimatvertrie- } \\
\text { benen und Entrechteten }\end{array}$ & EDC & $\begin{array}{l}\text { European Defense } \\
\text { Community }\end{array}$ \\
\hline & Bundeskanzler & EGKS & $\begin{array}{l}\text { Europäische Gemeinschaft } \\
\text { für Kohle und Stahl }\end{array}$ \\
\hline BMF & $\begin{array}{l}\text { Bundesminister/ium der } \\
\text { Finanzen }\end{array}$ & EPU & European Payments Union \\
\hline BMWi & $\begin{array}{l}\text { Bundesminister/ium für } \\
\text { Wirtschaft }\end{array}$ & EUCOM & $\begin{array}{l}\text { European Command, } \\
\text { United States Army }\end{array}$ \\
\hline BSt. & Besatzungsstatut & EVG & $\begin{array}{l}\text { Europäische Verteidigungs- } \\
\text { gemeinschaft }\end{array}$ \\
\hline $\mathrm{BvD}$ & $\begin{array}{l}\text { Bund vertriebener } \\
\text { Deutscher }\end{array}$ & EZU & Europäische \\
\hline CDU & $\begin{array}{l}\text { Christlich-Demokratische } \\
\text { Union Deutschlands }\end{array}$ & FDP & $\begin{array}{l}\text { Freie Demokratische } \\
\text { Partei }\end{array}$ \\
\hline COCOM & Coordinating Committee & & \\
\hline ČSR & Československá Republika & FU & Föderalistische Union \\
\hline CSU & Christlich-Soziale Union & GATT & $\begin{array}{l}\text { General Agreement on } \\
\text { Tariffs and Trade }\end{array}$ \\
\hline $\mathrm{D}$ & (Ministerial-)Direktor & Geh./geh. & Geheim/geheim \\
\hline DDR & $\begin{array}{l}\text { Deutsche Demokratische } \\
\text { Republik }\end{array}$ & GG & Grundgesetz \\
\hline Del. & Delegation & GK & Generalkonsul \\
\hline $\mathrm{Dg}$ & (Ministerial-)Dirigent & GK I & Generalkonsul I. Klasse \\
\hline DGB & $\begin{array}{l}\text { Deutscher Gewerkschafts- } \\
\text { bund }\end{array}$ & GMBL & $\begin{array}{l}\text { Gemeinsames } \\
\text { Ministerialblatt der } \\
\text { Bundesministerien }\end{array}$ \\
\hline DHG & $\begin{array}{l}\text { Deutsche } \\
\text { Handelsgesellschaft }\end{array}$ & HaPol & $\begin{array}{l}\text { Handelspolitische } \\
\text { Abteilung }\end{array}$ \\
\hline DIHT & $\begin{array}{l}\text { Deutscher Industrie- und } \\
\text { Handelstag }\end{array}$ & HICOG & $\begin{array}{l}\text { United States High } \\
\text { Commissioner for Germany }\end{array}$ \\
\hline $\begin{array}{l}\text { DP } \\
\text { DPB }\end{array}$ & $\begin{array}{l}\text { Deutsche Partei } \\
\text { Deutsche Partei Bayern }\end{array}$ & IKRK & $\begin{array}{l}\text { Internationales Komitee } \\
\text { vom Roten Kreuz }\end{array}$ \\
\hline
\end{tabular}




\begin{tabular}{|c|c|c|c|}
\hline IRK & Internationales Rotes & RAF & Royal Air Force \\
\hline & & $\mathrm{RM}$ & Reichsmark \\
\hline IRO & $\begin{array}{l}\text { International Refugee } \\
\text { Organization }\end{array}$ & RR & Regierungsrat \\
\hline IWF & $\begin{array}{l}\text { Internationaler Währungs- } \\
\text { fonds }\end{array}$ & SACEUR & $\begin{array}{l}\text { Supreme Allied } \\
\text { Commander Europe }\end{array}$ \\
\hline KPD & $\begin{array}{l}\text { Kommunistische Partei } \\
\text { Deutschlands }\end{array}$ & SCAP & $\begin{array}{l}\text { Supreme Commander for } \\
\text { the Allied Powers in Japan }\end{array}$ \\
\hline KPdSU & $\begin{array}{l}\text { Kommunistische Partei der } \\
\text { Sowjetunion }\end{array}$ & SED & $\begin{array}{l}\text { Sozialistische Einheits- } \\
\text { partei Deutschlands }\end{array}$ \\
\hline KPF & $\begin{array}{l}\text { Kommunistische Partei } \\
\text { Frankreichs }\end{array}$ & SHAPE & $\begin{array}{l}\text { Supreme Headquarters } \\
\text { Allied Powers Europe }\end{array}$ \\
\hline LR & Legationsrat & SIM & Surplus Incentive Material \\
\hline LR I & Legationsrat I. Klasse & SPD & $\begin{array}{l}\text { Sozialdemokratische Partei } \\
\text { Deutschlands }\end{array}$ \\
\hline MB & Ministerbüro & SRP & Sozialistische Reichspartei \\
\hline MD & Ministerialdirektor & SS & Schutzstaffel \\
\hline MDg & Ministerialdirigent & StEG & Staatliche Erfassungs- \\
\hline Mio. & Million/en & & Gesellschaft für öffent- \\
\hline MR & Ministerialrat & & liches Gut \\
\hline Mrd. & Milliarde/n & StS & Staatssekretär \\
\hline MRP & $\begin{array}{l}\text { Mouvement Républicain } \\
\text { Populaire }\end{array}$ & SVZ & $\begin{array}{l}\text { Saarländische } \\
\text { Volkszeitung }\end{array}$ \\
\hline MSA & Mutual Security Agency & TCC & Temporary Council \\
\hline MSI & Movimento Sociale Italiano & & Committee \\
\hline NATO & North Atlantic Treaty & Tgb. Nr. & Tagebuch-Nummer \\
\hline NS & $\begin{array}{l}\text { Organization } \\
\text { Nationalsozialismus }\end{array}$ & UdSSR & $\begin{array}{l}\text { Union der Sozialistischen } \\
\text { Sowjetrepubliken }\end{array}$ \\
\hline NSDAP & $\begin{array}{l}\text { Nationalsozialistische } \\
\text { Deutsche Arbeiterpartei }\end{array}$ & UEF & $\begin{array}{l}\text { Union Européenne des } \\
\text { Fédéralistes }\end{array}$ \\
\hline OEEC & $\begin{array}{l}\text { Organisation for European } \\
\text { Economic Cooperation }\end{array}$ & UN & United Nations \\
\hline $\begin{array}{l}\text { ORR } \\
\mathrm{Pg}\end{array}$ & $\begin{array}{l}\text { Oberregierungsrat } \\
\text { Parteigenosse }\end{array}$ & UNESCO & $\begin{array}{l}\text { United Nations Educa- } \\
\text { tional, Scientific and } \\
\text { Cultural Organization }\end{array}$ \\
\hline PNM & $\begin{array}{l}\text { Partito Nazionale } \\
\text { Monarchico }\end{array}$ & UNO & $\begin{array}{l}\text { United Nations } \\
\text { Organization }\end{array}$ \\
\hline PSI & Partito Socialista Italiano & US & United States \\
\hline PSLI & $\begin{array}{l}\text { Partito Socialista } \\
\text { Lavoratori Italiani }\end{array}$ & USA & United States of America \\
\hline PS-SIIS & Partito Socialista Sezione & VDL & $\begin{array}{l}\text { Verband Deutscher } \\
\text { Landsmannschaften }\end{array}$ \\
\hline & Socialista & VLR & Vortragender Legationsrat \\
\hline PSU & Partito Socialista Unitario & VS & Verschlußsache \\
\hline
\end{tabular}

